\title{
Boosting Self-interaction of Molecular Vibrations under Ultra-strong Coupling Condition
}

\author{
Akhila Kadyan ${ }^{1}$, Anil Shaji ${ }^{2} *$ and Jino George ${ }^{1 *}$ \\ ${ }^{1}$ Department of Chemical Sciences, Indian Institute of Science Education and Research (IISER) \\ Mohali, Punjab-140306, India. ${ }^{2}$ School of Physics, Indian Institute of Science Education and \\ Research (IISER) Thiruvananthapuram, Vithura, Kerala-695551, India.
}

\begin{abstract}
:
In this letter, we investigated the modification of oscillator strength of an asymmetric stretching band of $\mathrm{CS}_{2}$ by strong coupling to an infrared cavity photon. This is achieved by placing liquid $\mathrm{CS}_{2}$ in a Fabry-Perot resonator and tune the cavity mode position to match with the molecular vibrational transition. Ultra-strong coupling improves the self-interaction of transition dipoles of asymmetric stretching band of $\mathrm{CS}_{2}$ that resulted in an increase of its own oscillator strength. We experimentally proved this by taking the area ratio of asymmetric stretching and combination band by selectively coupling the former one. A non-linear increase in the oscillator strength of the asymmetric stretching band is observed upon varying the coupling strength. This is explained by a quantum mechanical model that predicts quadratic behavior under ultra-strong coupling
\end{abstract}


condition. These findings will set up a new paradigm for understanding chemical reaction modification by vacuum field coupling.

\section{TOC GRAPHICS}

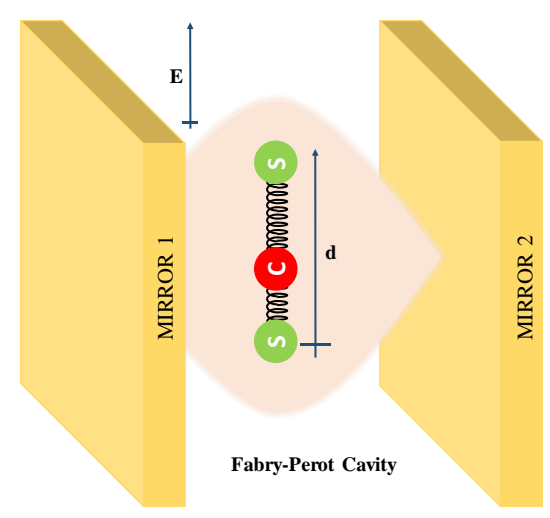

KEYWORDS: Vibrational spectroscopy, Light-matter strong coupling, Vibro-polaritonic state, Combination bands, Dipole-dipole interaction. 
Vibrational strong coupling (VSC) offers a new way of controlling the chemical and physical properties of the molecules and materials. $\left[{ }^{1},{ }^{2}\right]$ It involves the coupling of a selected vibrational state to an infrared cavity photon that undergoes photon exchange between the excited state of the molecule to the cavity field. This exchange process leads to a strong interaction within the system. Depending upon the strength of the interaction, energy levels get reshuffled to an extent that physically modifies their properties. $\left[{ }^{3}\right]$ These newly formed hybrid states are called vibropolaritonic states which have both molecule-like and photon-like behavior. $\left[{ }^{1},{ }^{5},{ }^{6}\right]$ In some cases the splitting energy is more than $10 \%$ of the fundamental transition, resulting in a dramatic change of their chemical and physical behavior. $\left[{ }^{7},{ }^{8}, 9\right]$ For example, chemical reactions can be modified by coupling to vacuum fluctuations. Recently, there are many attempts to prove this concept by placing reacting molecules in an optical resonator and probing their reaction kinetics. $\left[{ }^{10},{ }^{11},{ }^{12},{ }^{13},{ }^{14}\right]$ Surprisingly, some of the reactions got deaccelerated and some others catalyzed depending upon the reaction conditions. $\left[{ }^{10},{ }^{12},{ }^{14}\right]$ Recent experiments suggest that symmetry has a major role in controlling chemical reactions under VSC condition. $\left[{ }^{15}, 16\right]$ Very recently, co-operative VSC was also introduced to control chemistry. $\left[{ }^{12},{ }^{14}\right]$ This offers another advantage that solvent can be used to activate a chemical reaction pathway if the vibrational level of the solvent overlaps with reactant molecules. $\left[{ }^{12}\right] \mathrm{VSC}$ can be also used as a spectroscopic tool to understand a complicated reaction mechanism in some of the (bio)chemical reactions. $\left[{ }^{14}\right]$ There are many theoretical approaches available in the literature that covers from $\mathrm{ON}$ resonance effect to equilibrium theory.[ ${ }^{17},{ }^{18},{ }^{19},{ }^{20},{ }^{21}$ $\left.{ }^{22}{ }^{23}\right]$ Many of the experimental and theoretical predictions are so selective and fail to provide a clear understanding on the influence of VSC on chemical reaction. Even-though, VSC modifies the reaction rates, a universal model is yet to be established to understand the basic mechanism. 
Small molecule VSC is the only way to solve this conundrum as it can give a simplified approach in terms of spectral characterization. In this direction, there are few approaches so far reported in the literature. $\left[{ }^{7}\right]$ The first attempt was to solve the spectroscopic signature of $\mathrm{Fe}(\mathrm{CO})_{5}$ and $\mathrm{CS}_{2}$ molecules under ultra-vibrational strong coupling (USC) conditions. $\left[^{7}\right]$ There the self-interactions perturb the $\mathrm{N}$-dependent collective behavior that normally has been observed in the strongly coupled system. Along with this interesting finding, a clear polaritonic band gap opening is also seen experimentally that implies a complete modification of the absolute zero-point energy of the coupled system. On the theoretical side, a QE-DFT method was proposed by A. Rubio and coworkers using 1-D hydrogen atom model to predict the complicated polaritonic state potential energy surface (PES). $\left[{ }^{18}\right]$ Here, calculations show that the ground state energy shift along with a collapse of the PES leads to a complete reshuffling of the energy levels. Recent studies show clear entropy reordering due to collective interaction in strongly coupled systems. $\left[{ }^{24}\right]$ On the other hand, A. Nitzan and co-workers proposed an equilibrium consideration based on transition state theory. $\left[{ }^{20,25}\right]$ An extended ab-initio method was also introduced to study small molecule reaction dynamics. $\left[{ }^{26}\right]$ On the experimental side, Huira et.al. demonstrated that VSC of water molecules can catalyze chemical reactions. $\left[{ }^{27}\right]$ Water strong coupling experiments were also conducted to control biomolecular reactions. $\left[{ }^{28}\right]$ All the theoretical and experimental findings urge the use of small molecule VSC studies to develop a microscopic model for understanding the mechanism of polaritonic chemistry.

$\mathrm{CS}_{2}$ in the liquid state is a well-studied system by spectroscopists. $\left[{ }^{29}, 30,{ }^{31}\right]$ It contains 4 normal modes of vibrations in which the bending mode (010) is doubly degenerate. $\left[{ }^{29}\right]$ Other important vibrational states are asymmetric stretching (001) and symmetric stretching (100) bands as shown in Figure 1a. Out of these two, (001) is IR active and has very high oscillator strength. $\left[{ }^{29},{ }^{31}\right]$ For 
pure $\mathrm{CS}_{2}$, various combination and difference bands are observed with a reasonably good population. $\left[{ }^{31}\right]$ In the solution phase, $(101)$ is one of the highly populated combination bands and an area ratio of 45 is observed between (001) and (101). Here, these bands are fitted to multiLorentzian envelope and compared in each experimental case (figure S2). The area ratio is a constant value with respect to $\mathrm{CS}_{2}$ concentration in any of the solvent system. (001) is normally very strong and appears at $1510 \mathrm{~cm}^{-1}$, whereas, (101) is at $2155 \mathrm{~cm}^{-1}$ with a structured feature as shown in figure 1c. The structuring of (101) is specifically due to $\mathrm{C}^{32} \mathrm{~S}^{34} \mathrm{~S}$ isotopes and hot band transitions. $\left[{ }^{31}\right]$

Fabry-Perot (FP) cavities are fabricated by placing two parallel mirrors separated by a mylar spacer. $\mathrm{Au}$ mirrors $(10 \mathrm{~nm})$ are sputtered onto IR transparent $\mathrm{BaF}_{2}$ windows which serve as components of the flow cell cavity as shown in figure $1 \mathrm{~b}$. Here, in the present study, $\mathrm{CS}_{2}$ is injected (45 v\% in $\mathrm{CCl}_{4}$ ) in an $\mathrm{FP}$ cavity and the position of the mirrors are adjusted to fine-tune the $\mathrm{ON}$ resonance condition for coupling (001). This resulted in the splitting of (001) into two vibropolaritonic states with Rabi splitting energy of $\sim 180 \mathrm{~cm}^{-1}$ (figure 1c). The full width-half maximum (FWHM) of the (001) band and the empty cavity mode is $43 \mathrm{~cm}^{-1}\left(\Gamma_{\mathrm{m}}\right)$ and $23 \mathrm{~cm}^{-1}\left(\Gamma_{\mathrm{c}}\right)$, respectively. It is clear that vibro-polaritonic states formed at $\mathrm{ON}$ resonance condition have FWHM $\left(\Gamma_{\mathrm{p}+}\right.$ and $\Gamma_{\mathrm{p}-} ; 12 \mathrm{~cm}^{-1}$ and $14 \mathrm{~cm}^{-1}$, respectively) much smaller than the empty cavity mode and the bare molecule (figure 1c). At the same time, (101) is weakly coupled to one of the higherorder cavity modes, allowing us to trace its identity. Very interestingly, USC of (001) causes a reasonable increase in the area ratio of asymmetric to combination bands at ON resonance condition. 
Further, strong coupling experiments are conducted by injecting a neat solution of $\mathrm{CS}_{2}$ coupled to $10^{\text {th }}$ mode of the cavity and the original (001) transition is now split into multiple vibro-polaritonic states as shown in figure 2a. Mode folding occurs due to large refractive index modulation around (001) that brings the nearby cavity modes to couple OFF resonantly to the fundamental transition. $\left[{ }^{7}\right]$ The splitting energy of the $\mathrm{ON}$ resonant states (denoted as $\mathrm{P}^{+}{ }_{\mathrm{n}(001)}$ and $\mathrm{P}_{\mathrm{n}(001)}^{-}$) is $\sim 280$ $\mathrm{cm}^{-1}$ suggesting that the system is in USC condition. Here, the fundamental (001) transition is split more than $18 \%$, cause a large perturbation in the coupled system. At the same time, $\Gamma_{\mathrm{p}+}$ and $\Gamma_{\mathrm{p}-}$ are 10 and $12 \mathrm{~cm}^{-1}$, respectively, creating a much more stable vibro-polaritonic envelope (figure 2a). Under USC of (001), Rabi splitting energy is higher than predicted by TMM simulation. Hence, TMM fitting is done carefully to match the experimental data for (001) transition. This is arbitrarily achieved by varying the oscillator strength of the (001) transition on a multi-Lorentzian envelope of the uncoupled molecules (Section S3; figure S1). We used the same oscillator strength correction for (101) carefully analyzing the variation, assuming that the combination band strength is not affected under weak coupling regime (figure S2). This serves as a good internal reference to understand the physical effect of USC on (001) transition. Please note that (001) band position saturates in higher concentration of $\mathrm{CS}_{2}$ for our experimental condition (pathlength of $18 \mu \mathrm{m}$ ). Hence, we fitted the bare absorption of $\mathrm{CS}_{2}$ in $2 \mu \mathrm{m}$ pathlength to extract the area ratio (figure $\mathrm{S} 1$ and table $\mathrm{S} 1$ ). The area ratio of $\mathrm{CS}_{2}$ is $\sim 44.5$ in all the concentrations indicating the system obeys Beer's law throughout the non-cavity condition (figure S5). At ON resonance condition, TMM results suggest a deviation of more than $10 \%$ of splitting energy as compared to experimental data (blue trace; figure 2a). This observation is supported by the tuning experiments with respect to USC of the (001) transition. Changing the cavity mode resonance at (001) by slowly varying the pathlength leads to less deviation and eventually the same signature for weakly coupled (101) as 
OFF resonance situation is achieved (figure 2b). Here, all the other parameters are fixed except the pathlength of the cavity mode so that the $10^{\text {th }}$ mode undergoes USC with (001). Overall, USC of (001) causes a modification of its own oscillator strength that can be assessed through (101) of the $\mathrm{CS}_{2}$ molecule at $\mathrm{ON}$ resonance condition.

In the next step, we varied the concentration of the $\mathrm{CS}_{2}$ molecule and observed the effect of USC of (001) on the combination bands. Here, we used $\mathrm{CCl}_{4}$ as the co-solvent for dilution as the latter is transparent in the IR optical window. To understand the effect of coupling strength, $\mathrm{CCl}_{4}$ (refractive index; $\mathrm{n}=1.45)$ is added into $\mathrm{CS}_{2}(\mathrm{n}=1.60)$ in a proportion to match the criteria of having two different cavity modes couple to (001) and (101) simultaneously. Other than pure $\mathrm{CS}_{2}$, $85 \mathrm{v} \%, 45 \mathrm{v} \%$, and $25 \mathrm{v} \%$ are chosen for this experiment and the corresponding results are plotted in figure 3. There is no variation in the area ratio is observed for the non-cavity condition. Whereas, the system shows a clear variation in the area ratio as its coupling strength changes from low to high concentration of $\mathrm{CS}_{2}$ molecules. For the coupled system the area ratio changes non-linearly, at the same time, the Rabi splitting of (001) and (101) follows linearly with the square root of concentration (inset of figure 3). This shows that some non-linear interactions are taking place while coupling the (001) band to the cavity field.

For understanding this we used a simple quantum mechanical model considering $\mathrm{CS}_{2}$ molecules whose axis is perpendicular to the axis of the cavity and can only couple to the transverse oscillations of the cavity field. The asymmetric stretching mode for each molecule is characterized by the dimensionless coordinate,

$$
\xi=\sqrt{\frac{\mu \omega_{3}}{\hbar}} z ; \text { where } z=z_{3}-\frac{z_{1}+z_{2}}{2} \text {. }
$$


In the equation above, $z_{1}, z_{2}$ and $z_{3}$ are the coordinates along the molecular axis of the two sulfur and one carbon atom respectively measured relative to the center of mass of the two sulfur atoms. $\left[{ }^{32},{ }^{33}\right]$ Corresponding to an asymmetric displacement $z$ of the carbon atom along the molecular axis, the dipole moment operator of the molecule is,

$$
\vec{p}(0)=\left[\frac{d\langle\vec{p}\rangle}{d z}\right]_{z_{0}} z=\left[\frac{d\langle\vec{p}\rangle}{d z}\right]_{z_{0}} \sqrt{\frac{\hbar}{\mu \omega_{3}}} \xi=\left[\frac{d\langle\vec{p}\rangle}{d z}\right]_{z_{0}} \sqrt{\frac{\hbar}{2 \mu \omega_{3}}}\left(a_{\xi}+a_{\xi}^{\dagger}\right),
$$

where $a_{\xi}$ and $a_{\xi}^{\dagger}$ are the annihilation and creation operators corresponding to the asymmetric stretching mode oscillations. The collective polarization of the $n$ molecules is,

$$
\vec{P}(0)=\sum_{j=1}^{n} \vec{p}(0)=\left[\frac{d\langle\vec{p}\rangle}{d z}\right]_{z_{0}} \sqrt{\frac{\hbar}{2 \mu \omega_{3}}} \sum_{j=1}^{n}\left(a_{\xi j}+a_{\xi j}^{\dagger}\right)
$$

The cavity-molecule coupling is described by the Hamiltonian,

$$
H_{C M}=\vec{E} \cdot \vec{P}=\hbar \Omega \sum_{j=1}^{n}\left(a_{c}+a_{c}^{\dagger}\right)\left(a_{\xi j}+a_{\xi j}^{\dagger}\right) ; \quad \Omega=\left[\frac{d\langle\vec{p}\rangle}{d z}\right]_{z_{0}} \frac{1}{\sqrt{2 \epsilon_{0} V}}
$$

Where $a_{c}$ and $a_{c}^{\dagger}$ are the annihilation and creation operators of the relevant cavity mode, $\epsilon_{0}$ is the permittivity of free space and $V$ is the mode volume within which the molecules interacting with the mode are located.

In the USC regime, this interaction between the cavity and the molecules cannot be treated perturbatively. Powerful numerical approaches have been developed to compute the spectra and other relevant parameters in the presence of very strong coupling including the QE-DFT 
method. $\left[{ }^{18}\right]$ However here we can take a much more direct and elementary approach since we are interested only in the vibrational motion of the molecules.

The $\vec{E} \cdot \vec{P}$ (light-matter) interaction term is proportional to $\xi$. If a term that is linear in the coordinate is added to the quantum harmonic oscillator Hamiltonian, the problem can still be solved exactly by transforming the Hamiltonian into one for an oscillator with a shifted equilibrium position plus a constant energy shift. The shifted equilibrium of the asymmetric stretching mode indicates the induced dipole moment of the molecule due to the presence of the cavity. The induced dipoles of the $n$ molecules that oscillate resonantly and coherently with the oscillations of the cavity field, interact with each other. For weak coupling this interaction is an insignificant higherorder effect. However, for USC, the dipole-dipole interactions lead to significant collective, nonlinear effects. The Hamiltonian for this interaction is,

$$
H_{M M}=\frac{1}{2 \epsilon_{0} V} \vec{P} \cdot \vec{P}=\hbar \frac{\Omega^{2}}{\omega_{3}} \sum_{j . k}\left(a_{\xi j}+a_{\xi j}^{\dagger}\right)\left(a_{\xi k}+a_{\xi k}^{\dagger}\right),
$$

where $\omega_{3}$ is the natural frequency of the asymmetric stretching mode.

In the USC regime, the dipole-dipole coupling also has to be treated non-perturbatively. The corresponding Hamiltonian, $H_{M M}$ is quadratic in $\xi$. Therefore, the dipole-dipole interaction can be incorporated into the harmonic oscillator Hamiltonian as a simple shift in the frequency. Adding a term to the harmonic oscillator Hamiltonian of the form $\frac{1}{2} \hbar K \xi^{2}$, with $K$ being a constant, effectively shifts the frequency of the oscillator by $K$. Finding the strengths of the collective dipolecavity coupling $H_{C M}$ and the dipole-dipole coupling $H_{M M}$ will therefore allow us to identify the 
shift in the equilibrium position as well as the shift in frequency of the asymmetric stretching mode.

A detailed analysis of the molecule-cavity system shows that the effective coupling constant in $H_{C M}$ (Rabi splitting) is given by $\hbar \Omega_{\mathrm{R}}$ where the Rabi frequency is given by $\Omega_{R}=\sqrt{n} \Omega$. The effective strength of the dipole-dipole coupling is given by $\left.\frac{\hbar \Omega_{R}^{2}}{\omega_{3}}=2 n \hbar \Omega^{2} / \omega_{3} \cdot{ }^{34}\right]$ Assuming that the number of molecules $n$ oriented perpendicular to the cavity axis and located within the mode volume of the resonant vacuum mode of the cavity is proportional to the concentration, $C$, of $\mathrm{CS}_{2}$ within the cavity, we expect two detectable collective effects in the USC regime. The first is a concentration dependent Rabi shift which is proportional to $\sqrt{n} \sim \sqrt{C}$ and the second is the shift in the frequency of the asymmetric stretching mode which is proportional to $C$. It is not easy to detect the shift in the bare frequency $\omega_{3}$ because of the Rabi splitting that is larger than the shift by a factor of $\omega_{3}$.

Using perturbation theory to find the transition rate for the normally dipole forbidden transition to the combination band, made possible by the anharmonicity of the molecular potential (See Supporting information for details), yields an expression for the measured area ratio as,

$$
A=\Gamma \frac{\omega_{3}}{\omega_{1}+\omega_{3}}
$$

Where $\Gamma$ is effectively a constant despite a very weak dependence on $\omega_{1}$ and $\omega_{3}$. We have extracted out of the expression for $A$, the dominant dependence on $\omega_{3}$.

In the USC regime, we expect $\omega_{3} \rightarrow \omega_{3}+2 n \frac{\Omega^{2}}{\omega_{3}}$, which we approximate as $\omega_{3}+\kappa n$. The $\omega_{3}$ appearing in the numerator of the expression for $A$ corresponds to the (000) to (001) transition 
while that appearing in the denominator corresponds to the (000) to (101) transition. Since the change in the denominator also depends on the admixture of (001) in the combination band, we write the dependence on the denominator on the concentration with an additional constant factor as $\omega_{1}+\omega_{3} \rightarrow \omega_{1}+\omega_{3}+\eta \kappa n$. Assuming that $n$ is proportional to the concentration, $C$, of CS2 as $n=\gamma C$ we obtain the following form for the expected relation between the measured area ratio and the concentration under USC conditions,

$$
A=A \frac{\omega_{3}}{\omega_{1}+\omega_{3}} \times \frac{1+C \gamma \kappa / \omega_{3}}{1+C \gamma \eta \kappa /\left(\omega_{1}+\omega_{3}\right)}=A_{0} \frac{1+k_{1} C}{1+k_{2} C^{\prime}}
$$

where $A_{0}$ is the area ratio in the absence of cavity coupling. Figure 3 shows a two-parameter fit (QM fitting) of the expression above for the observed changes in the area ratio. The fitted values of the parameters are $k_{1}=0.0185673$ and $k_{2}=0.00638233$ giving $\eta \sim 1 / 3$, indicating that there is approximately $33 \%$ admixture of the (001) band in the (101) combination band. We observe that the expression for the area ratio and the measured data are in very good agreement indicating that the system is indeed in the USC regime and the simple model phrased in terms of a quantum harmonic oscillator not only gives a clear picture of the nature of the interaction between the cavity and the molecules, it also yields a quantitative understanding of the same.

In conclusion, a clear boost in the self-interaction of vibrational transition dipole in $\mathrm{CS}_{2}$ molecules is observed under USC condition. Here, the non-linear change in the self-interaction is proved both experimentally and theoretically. Recent experiments on the non-linear behavior in the rate enhancement mediated through the polaritonic state may be triggered through this mechanism. $\left[{ }^{10}\right.$, ${ }^{12}$ ] However, the complexity of organic chemical reactions won't allow us to measure the relation between the rate enhancement and cavity mediated self-interaction in strongly coupled systems. 


\section{ASSOCIATED CONTENT}

Supporting Information. Details of materials and methods, preparation of cavity and non-cavity, IR spectrum of $\mathrm{CS}_{2}, \mathrm{TMM}$ simulations and area ratio calculations, area ratio for $\mathrm{ON}$ resonance cavity, tuning experiment, and details of the theoretical development are given in the supporting information.

\section{AUTHOR INFORMATION}

Corresponding Authors: *jgeorge@iisermohali.ac.in; shaji@iisertvm.ac.in

\section{Notes}

The authors declare no competing financial interests.

\section{ACKNOWLEDGMENT}

J.G. would like to acknowledge DST-SERB, Core Research Grant (EMR/2017/003455), and MHRD-Scheme for Transformational and Advanced Research in Sciences (STARS 175/2019) for financial support. A.S. acknowledges the support of DST-SERB through grant no. EMR/2016/007221 and the QuEST program of DST through project No. Q113 under Theme 4. A.K. thank IISER Mohali for the fellowship. Dr. Atef Shalabney, Braude Collge, Israel is thanked for teaching us TMM simulation.

\section{REFERENCES}

1. Ebbesen, T. W., Hybrid Light-Matter States in a Molecular and Material Science Perspective. Accounts of chemical research 2016, 49 (11), 2403-2412.

2. Herrera, F.; Owrutsky, J., Molecular polaritons for controlling chemistry with quantum optics. The Journal of Chemical Physics 2020, 152 (10), 100902. 
3. Kaluzny, Y.; Goy, P.; Gross, M.; Raimond, J. M.; Haroche, S., Observation of Self-Induced Rabi Oscillations in Two-Level Atoms Excited Inside a Resonant Cavity: The Ringing Regime of Superradiance. Physical Review Letters 1983, 51 (13), 1175-1178.

4. Long, J. P.; Simpkins, B. S., Coherent Coupling between a Molecular Vibration and FabryPerot Optical Cavity to Give Hybridized States in the Strong Coupling Limit. ACS Photonics 2015, $2(1), 130-136$.

5. Dunkelberger, A. D.; Spann, B. T.; Fears, K. P.; Simpkins, B. S.; Owrutsky, J. C., Modified relaxation dynamics and coherent energy exchange in coupled vibration-cavity polaritons. Nature Communications 2016, 7 (1), 13504.

6. Xiang, B.; Ribeiro, R. F.; Du, M.; Chen, L.; Yang, Z.; Wang, J.; Yuen-Zhou, J.; Xiong, W., Intermolecular vibrational energy transfer enabled by microcavity strong light-matter coupling. Science 2020, 368 (6491), 665.

7. George, J.; Chervy, T.; Shalabney, A.; Devaux, E.; Hiura, H.; Genet, C.; Ebbesen, T. W., Multiple Rabi Splittings under Ultrastrong Vibrational Coupling. Physical review letters 2016, $117(15), 153601$.

8. Shalabney, A.; George, J.; Hutchison, J.; Pupillo, G.; Genet, C.; Ebbesen, T. W., Coherent coupling of molecular resonators with a microcavity mode. Nature communications $\mathbf{2 0 1 5 , 6 , 5 9 8 1 . ~}$

9. Damari, R.; Weinberg, O.; Krotkov, D.; Demina, N.; Akulov, K.; Golombek, A.; Schwartz, T.; Fleischer, S., Strong coupling of collective intermolecular vibrations in organic materials at terahertz frequencies. Nature Communications 2019, 10 (1), 3248.

10. Thomas, A.; George, J.; Shalabney, A.; Dryzhakov, M.; Varma, S. J.; Moran, J.; Chervy, T.; Zhong, X.; Devaux, E.; Genet, C.; Hutchison, J. A.; Ebbesen, T. W., Ground-State Chemical Reactivity under Vibrational Coupling to the Vacuum Electromagnetic Field. Angewandte Chemie 2016, 55 (38), 11462-6.

11. Thomas, A.; Lethuillier-Karl, L.; Nagarajan, K.; Vergauwe, R. M. A.; George, J.; Chervy, T.; Shalabney, A.; Devaux, E.; Genet, C.; Moran, J.; Ebbesen, T. W., Tilting a ground-state reactivity landscape by vibrational strong coupling. Science 2019, 363 (6427), 615-619.

12. Lather, J.; Bhatt, P.; Thomas, A.; Ebbesen, T. W.; George, J., Cavity Catalysis by Cooperative Vibrational Strong Coupling of Reactant and Solvent Molecules. Angewandte Chemie International Edition 2019, 58 (31), 10635-10638. 
13. Hirai, K.; Takeda, R.; Hutchison, J. A.; Uji-i, H., Modulation of Prins Cyclization by Vibrational Strong Coupling. Angewandte Chemie International Edition 2020, 59 (13), 53325335.

14. Lather, J.; George, J., Improving Enzyme Catalytic Efficiency by Co-operative Vibrational Strong Coupling of Water. The Journal of Physical Chemistry Letters 2021, 12 (1), 379-384.

15. Pang, Y.; Thomas, A.; Nagarajan, K.; Vergauwe, R. M. A.; Joseph, K.; Patrahau, B.; Wang, K.; Genet, C.; Ebbesen, T. W., On the Role of Symmetry in Vibrational Strong Coupling: The Case of Charge-Transfer Complexation. Angewandte Chemie International Edition 2020, 59 (26), 10436-10440.

16. Sau, A.; Nagarajan, K.; Patrahau, B.; Lethuillier-Karl, L.; Vergauwe, R. M. A.; Thomas, A.; Moran, J.; Genet, C.; Ebbesen, T. W., Modifying Woodward-Hoffmann Stereoselectivity Under Vibrational Strong Coupling. Angewandte Chemie International Edition 2021, 60, 1-7.

17. (a) Pino, J.-D.; Feist, J.; Garcia-Vidal, F. J., Quantum theory of collective strong coupling of molecular vibrations with a microcavity mode. New J Phys 2015, 17 (5), 053040; (b) Feist, J.; Galego, J.; Garcia-Vidal, F. J., Polaritonic Chemistry with Organic Molecules. ACS Photonics 2018, 5 (1), 205-216; (c) Galego, J.; Garcia-Vidal, F. J.; Feist, J., Many-Molecule Reaction Triggered by a Single Photon in Polaritonic Chemistry. Physical Review Letters 2017, 119 (13), 136001.

18. (a) Flick, J.; Ruggenthaler, M.; Appel, H.; Rubio, A., Atoms and molecules in cavities, from weak to strong coupling in quantum-electrodynamics (QED) chemistry. Proceedings of the National Academy of Sciences 2017, 114 (12), 3026-3034; (b) Ruggenthaler, M.; TancogneDejean, N.; Flick, J.; Appel, H.; Rubio, A., From a quantum-electrodynamical light-matter description to novel spectroscopies. Nature Reviews Chemistry 2018, 2, 0118.

19. (a) Herrera, F.; Spano, F. C., Cavity-Controlled Chemistry in Molecular Ensembles. Physical Review Letters 2016, 116 (23), 238301; (b) Herrera, F.; Spano, F. C., Theory of Nanoscale Organic Cavities: The Essential Role of Vibration-Photon Dressed States. ACS Photonics 2018, 5 (1), 65-79.

20. Li, T. E.; Subotnik, J. E.; Nitzan, A., Cavity molecular dynamics simulations of liquid water under vibrational ultrastrong coupling. Proceedings of the National Academy of Sciences 2020, 117, 18324-18331. 
21. Ribeiro, R. F.; Martinez-Martinez, L. A.; Du, M.; Campos-Gonzalez-Angulo, J.; YuenZhou, J., Polariton chemistry: controlling molecular dynamics with optical cavities. Chemical Science 2018, 9, 6325-6339

22. Campos-Gonzalez-Angulo, J. A.; Yuen-Zhou, J., Polaritonic normal modes in transition state theory. The Journal of Chemical Physics 2020, 152 (16), 161101.

23. Li, X.; Mandal, A.; Huo, P., Resonance Theory of Vibrational Strong Couplings in Polariton Chemistry. ChemrXiv 2020, https://doi.org/10.26434/chemrxiv.12915701.v1.

24. Scholes, G. D.; DelPo, C. A.; Kudisch, B., Entropy Reorders Polariton States. The Journal of Physical Chemistry Letters 2020, 11 (15), 6389-6395.

25. Li, T.; Nitzan, A.; Subotnik, J. E., On the origin of ground-state vacuum-field catalysis: Equilibrium consideration. The Journal of chemical physics 2020, 152 23, 234107.

26. Flick, J.; Narang, P., Cavity-Correlated Electron-Nuclear Dynamics from First Principles. Physical review letters 2018, 121 (11), 113002.

27. Hiura, H.; Shalabney, A.; George, J., Cavity Catalysis -Accelerating Reactions under Vibrational Strong Coupling-. ChemrXiv 2018, 10.26434/chemrxiv.7234721.v3.

28. Vergauwe, R. M. A.; Thomas, A.; Nagarajan, K.; Shalabney, A.; George, J.; Chervy, T.; Seidel, M.; Devaux, E.; Torbeev, V.; Ebbesen, T. W., Modification of Enzyme Activity by Vibrational Strong Coupling of Water. Angewandte Chemie 2019, 58 (43), 15324-15328.

29. Plyler, E. K.; Humphreys, C., Infrared absorption spectrum of carbon disulfide. J. Res. Natl. Bur. Stand. 1947, 39, 59-65.

30. Mendelsohn, R.; Monse, E. U., Vibrational spectroscopy of CS2: A physical chemistry experiment. Journal of Chemical Education 1981, 58 (7), 582.

31. Ribni Kar, S. V.; Putzić, O. S., The infrared spectrum of carbon disulphide in liquid and dissolved states: anharmonicity of the carbon disulphide molecule in the liquid. Spectrochimica Acta Part A: Molecular Spectroscopy 1973, 29 (2), 307-317.

32. Dennison, D. M., The Vibrational Levels of Linear Symmetrical Triatomic Molecules. Physical Review 1932, 41 (3), 304-312.

33. Dennison, D. M., The Infrared Spectra of Polyatomic Molecules Part I. Reviews of Modern Physics 1931, 3 (2), 280-345.

34. Ciuti, C.; Bastard, G.; Carusotto, I., Quantum vacuum properties of the intersubband cavity polariton field. Phys Rev B 2005, 72 (11), 115303. 


\section{Figures:}
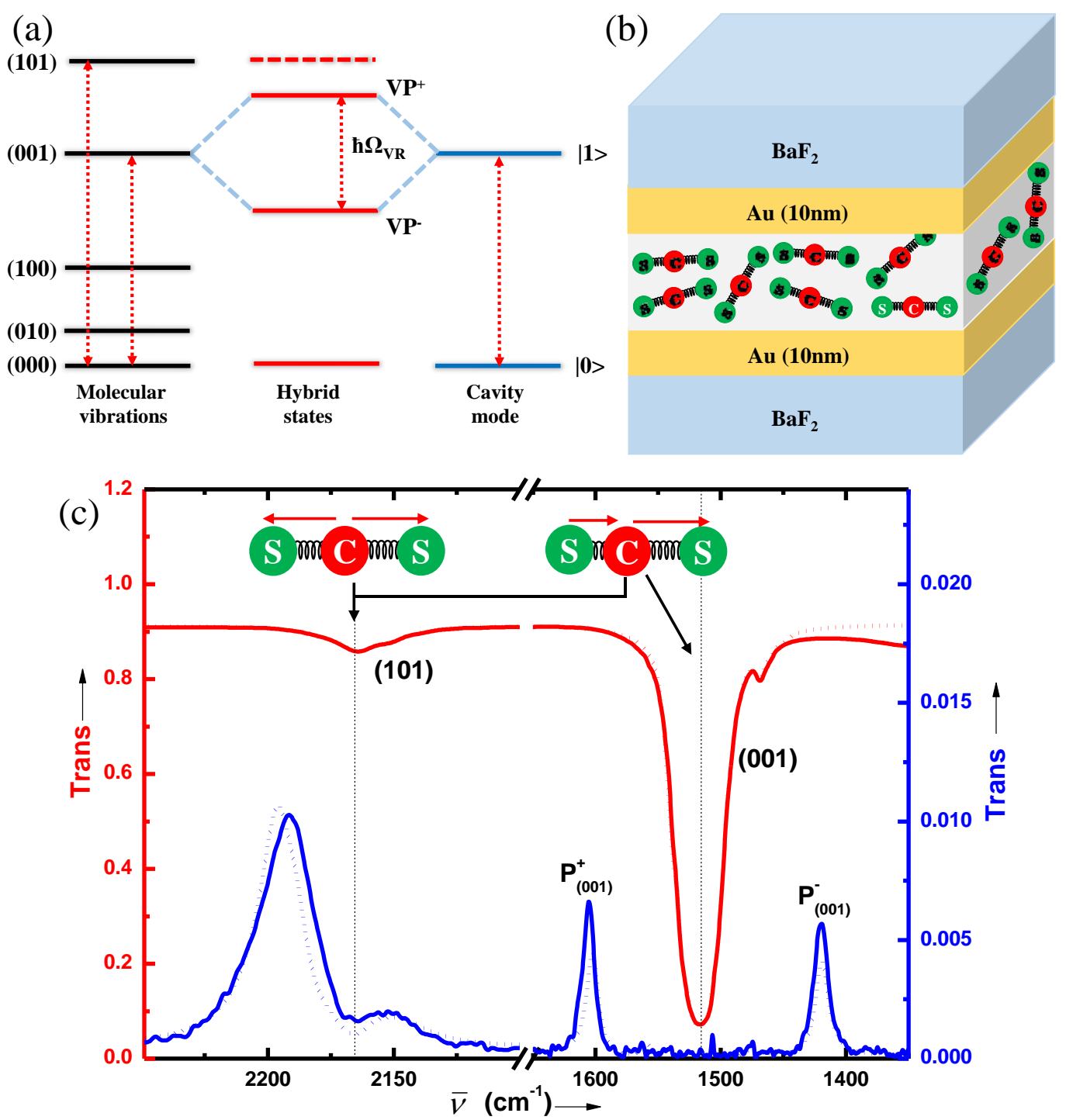

Figure 1. (a) Schematic illustration of VSC of $\mathrm{CS}_{2}$ molecule and (b) a Fabry-Perot cavity. (b) Infrared transmission spectra of non-cavity $(2 \mu \mathrm{m}$; red) and cavity (18 $\mu \mathrm{m}$; blue) by coupling of (001) band of $\mathrm{CS}_{2}(45 \mathrm{v} \%)$ in $\mathrm{CCl}_{4}$ solution and the corresponding $\mathrm{TMM}$ simulation (dotted lines). 
(a)

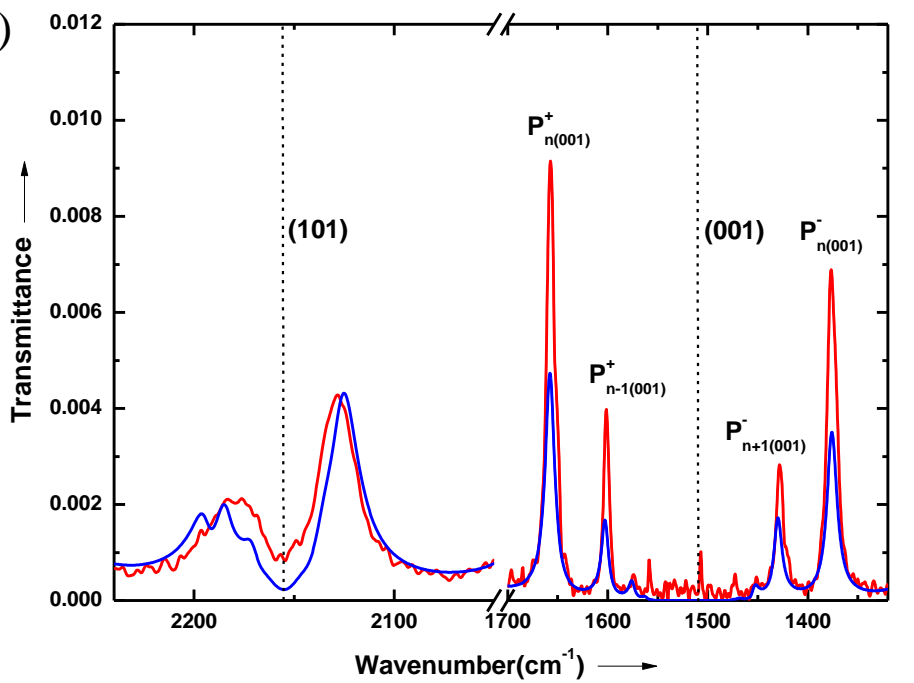

(b)

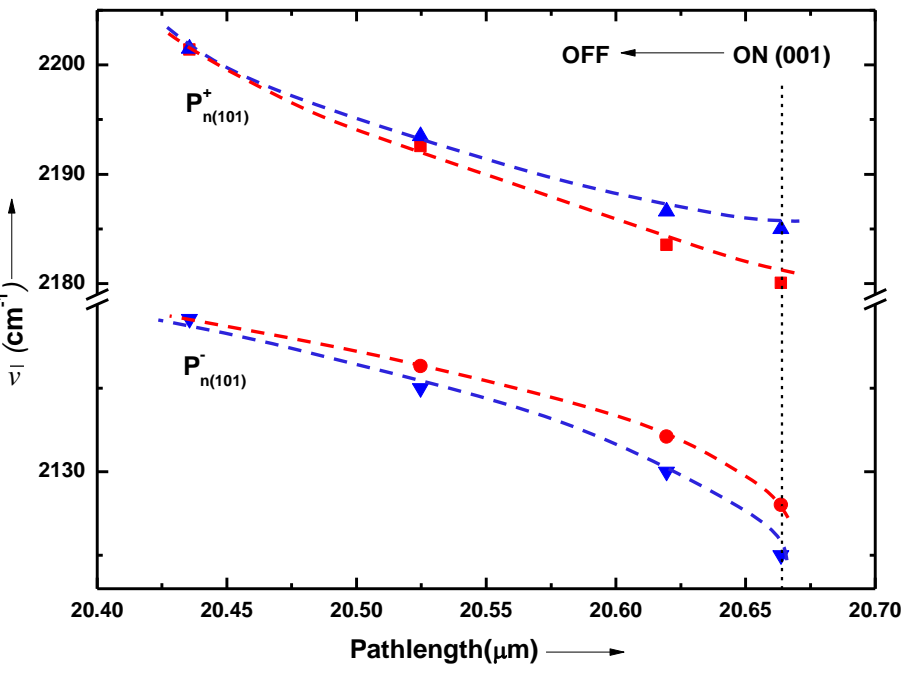

Figure 2. (a) IR transmission spectra of USC of pure $\mathrm{CS}_{2}$ (red) to $10^{\text {th }}$ mode of an FP cavity and the corresponding TMM (dotted red line) simulation; (b) experimental splitting energy of (101) band (red) and the corresponding deviation in TMM simulation (blue) [dotted lines are guide to eye]. 


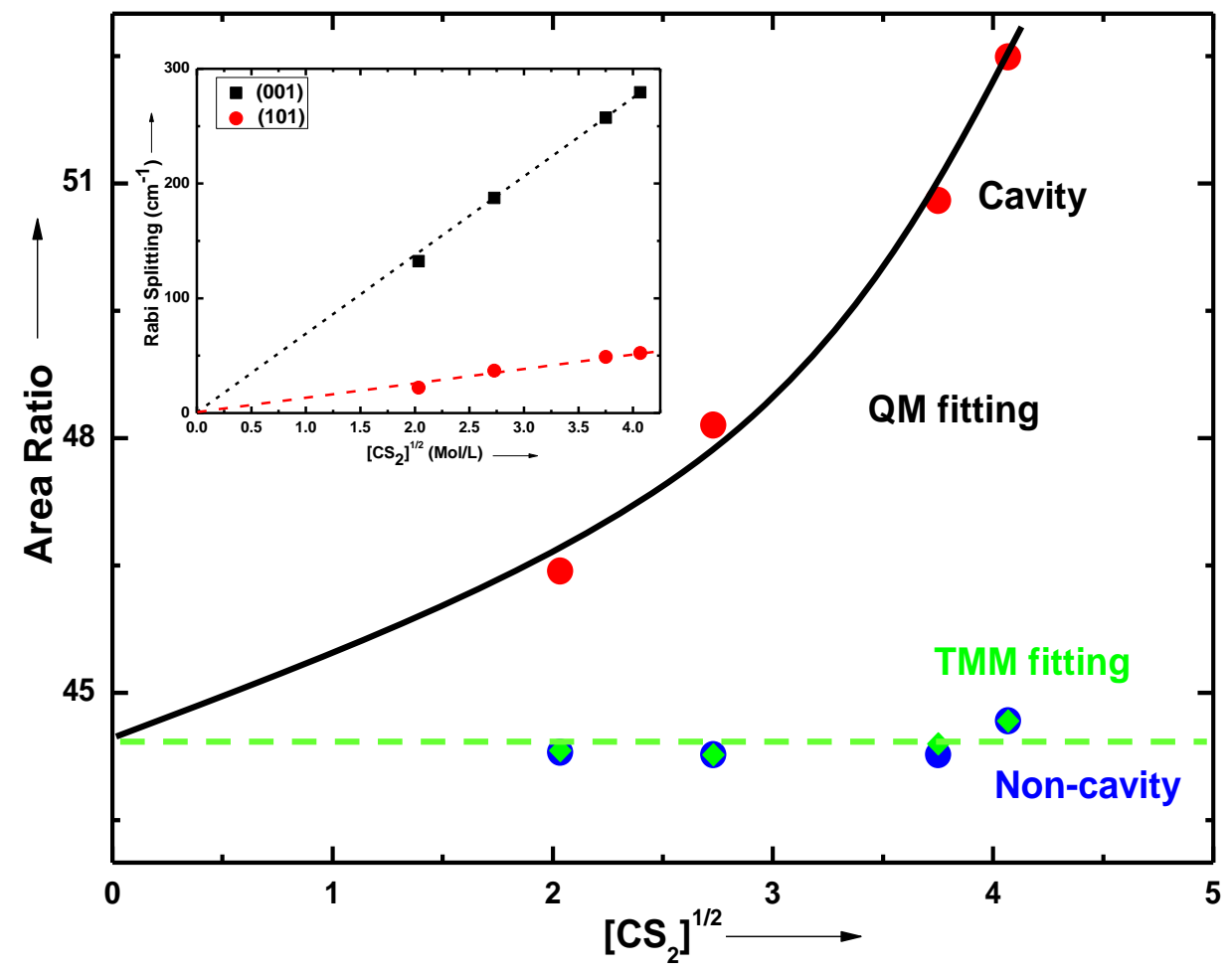

Figure 3. Plot of area ratio as a function of $\mathrm{CS}_{2}$ concentration in a non-cavity (blue) and under USC at ON resonance (red) and the corresponding TMM fitting (green). Black line is the quantum harmonic oscillator 2-parameter fitting (QM fitting). 


\section{Supporting Information}

\section{Contents}

1 Materials and Methods: $\quad 2$

2 Preparation of cavity and non-cavity cells: 3

3 IR spectrum of $\mathrm{CS}_{2}$, Transfer matrix method (TMM) fitting and area ratio calcula-

$\begin{array}{ll}\text { tions: } & 3\end{array}$

4 Area ratio for ON resonance cavity: 4

5 Area ratio in cavity tuning experiment: $\quad 7$

6 Beer-Lambert plot for Asymmetric stretching band 8

7 Theoretical expression for the area ratio 9

7.1 The linear triatomic molecule . . . . . . . . . . . . . . . . . 9

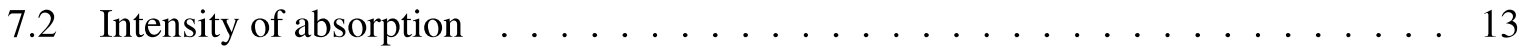

7.3 Physical states . . . . . . . . . . . . . . . . . 14

7.4 The $\mathrm{CS}_{2}$ Molecule $\ldots \ldots \ldots \ldots \ldots \ldots \ldots$

8 Ultra-strong coupling to the cavity $\quad 18$

\section{Materials and Methods:}

Carbon disulfide and Carbon tetrachloride used for the studies purchased from Sigma-Aldrich. The demountable flow cell, $\mathrm{BaF} 2$ substrates, and mylar spacers used for measurements purchased from Specac. 
The IR transmission spectrum of pure $\mathrm{CS}_{2}, 45 \%$ and $85 \% \mathrm{CS}_{2}$ solutions in $\mathrm{CCl}_{4}$ in both cavity and non-cavity recorded using the Fourier Transform Infrared (FT-IR) interferometer (C) Bruker model INVENIO). The spectra were recorded in transmission mode with a $2 \mathrm{~cm}^{-1}$ resolution under 16 scans.

\section{Preparation of cavity and non-cavity cells:}

The $2 \mathrm{~mm}$ thick $\mathrm{BaF}_{2}$ substrates were used as IR transmitting windows. The non-cavity measurements carried out by placing $\mathrm{BaF}_{2}$ substrates one above the other, without any spacer between the substrates, inside the demountable flow cell. Even in the absence of any spacer, a $2 \mu \mathrm{m}$ spacing created between the substrates due to their surface roughness. Then solutions of different concentrations of $\mathrm{CS}_{2}$ were injected to obtain their IR spectra. For the cavity measurements, $10 \mathrm{~nm}$ gold film sputtered on to the $\mathrm{BaF}_{2}$ substrates. A Fabry-Perot cavity is prepared by placing these gold-coated substrates separated by Mylar spacer in a demountable flow cell.

\section{IR spectrum of $\mathrm{CS}_{2}$, Transfer matrix method (TMM) fitting and area ratio calculations:}

Firstly, the IR transmission spectrum of pure $\mathrm{CS}_{2}$ recorded in a non-cavity. The spectrum consists of an inhomogeneously broadened asymmetric stretching band (001) at $1510 \mathrm{~cm}^{-1}$ and a combination band (101) of the asymmetric and symmetric stretch at $2155 \mathrm{~cm}^{-1}$ (Fig.S1(a)). TMM used to fit this spectrum using multi-Loretzians for fitting the asymmetric stretching band and combination band.

This transmission spectrum was converted into its corresponding absorption spectrum (Fig. S2(b)) to calculate the area under both the asymmetric stretching band and the combination band. With the help of OriginPro 9 software, the area under these curves was calculated. Using this method the area ratio of asymmetric to combination band was approximated to 44.6. Similar area 

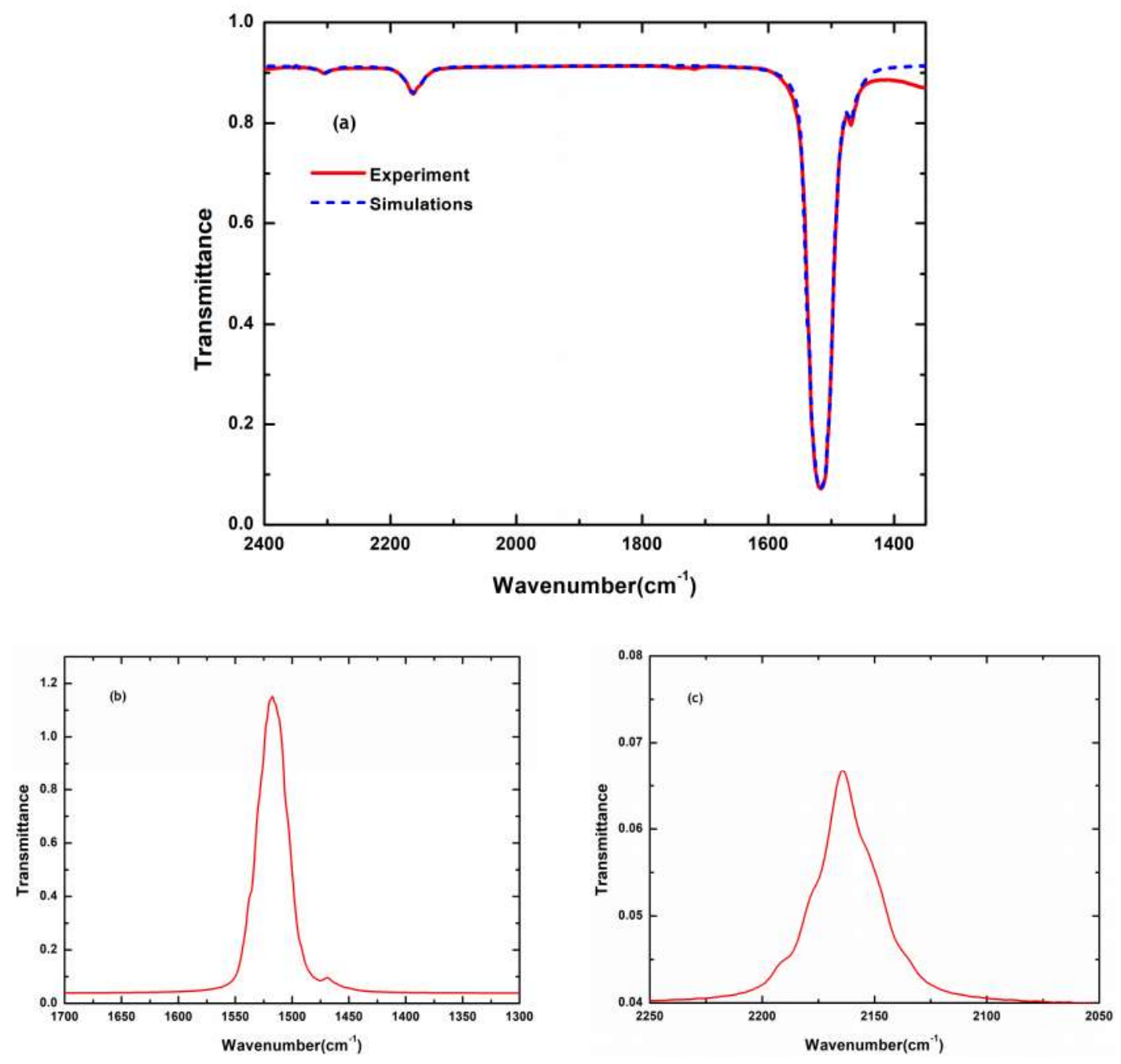

Figure S1: (a) Infrared transmission spectra of pure CS2 (red trace) and the corresponding TMM fitting (dotted black trace) outside the cavity. Infrared absorption spectra of pure $\mathrm{CS}_{2}$, (b) asymmetric stretching band (c) combination band.

ratio calculations were done for different concentrations of $\mathrm{CS}_{2}$ in $\mathrm{CCl}_{4}$ solutions, and results are summarized in Table 1, which shows area ratio remains approximately the same for all the concentrations of $\mathrm{CS}_{2}$ in the non-cavity conditions.

\section{Area ratio for ON resonance cavity:}

Here, we achieved the $\mathrm{ON}$ resonance condition by tuning the cavity mode position to resonate with the (001) mode of $\mathrm{CS}_{2}$ molecule. This will help us to understand the effect of strong coupling 
Table 1: Area ratio for different concentrations of $\mathrm{CS}_{2}$ in non-cavity conditions.

\begin{tabular}{|c|c|c|}
\hline Sr. No. & Concentration of CS CS $_{2}$ & Area Ratio \\
\hline \hline 1 & $25 \%(4.14 \mathrm{M})$ & 44.30 \\
\hline 2 & $45 \%(7.45 \mathrm{M})$ & 44.27 \\
\hline 3 & $85 \%(14.07 \mathrm{M})$ & 44.27 \\
\hline 4 & $100 \%(16.55 \mathrm{M})$ & 44.67 \\
\hline
\end{tabular}
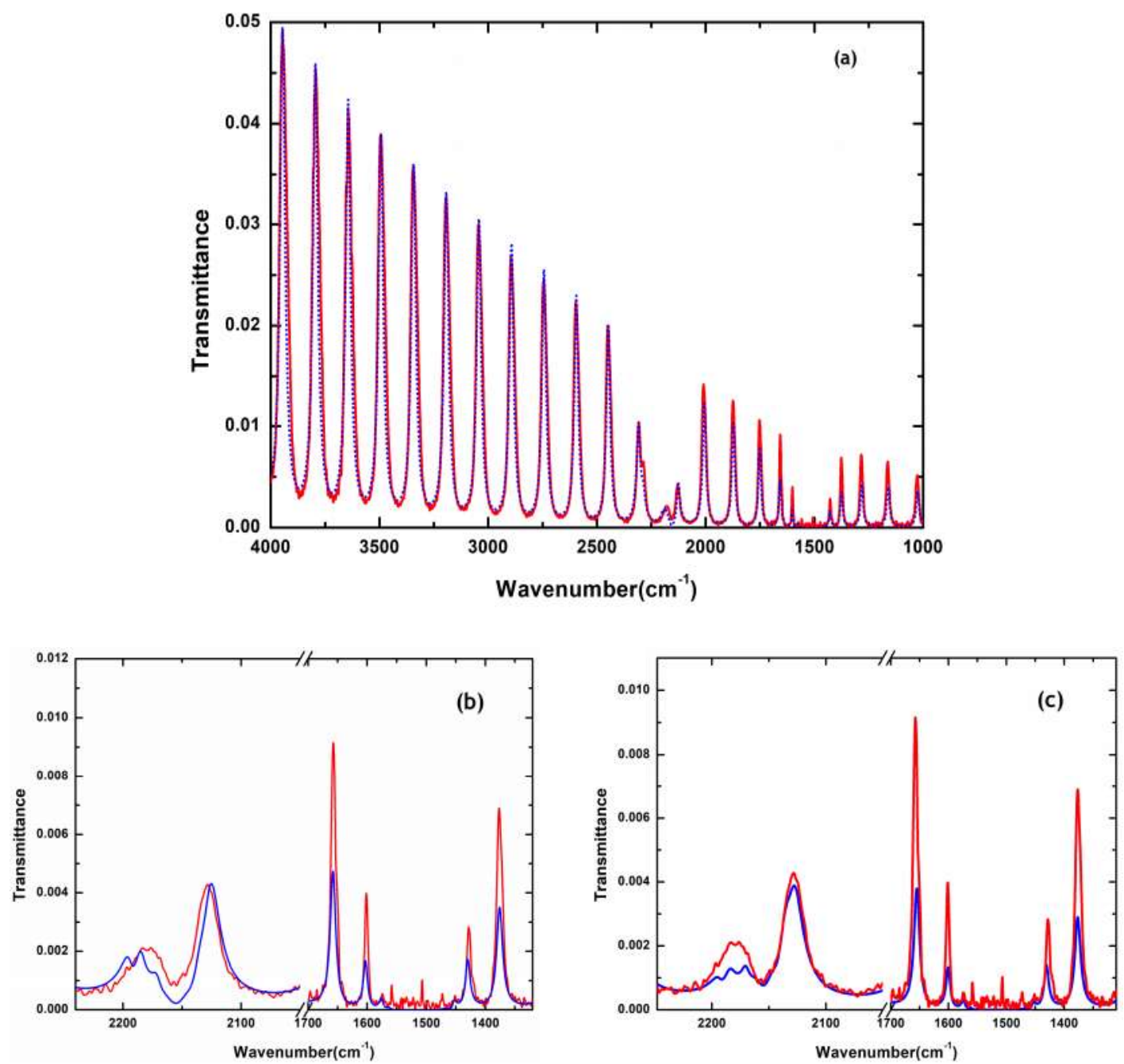

Figure $S 2$ : (a) IR transmission spectrum of Fabry-Perot $18 \mu \mathrm{m}$ cavity filled with pure $\mathrm{CS}_{2}$ (red: experiment; dotted blue: TMM) with $10^{\text {th }}$ cavity mode at $1512.33 \mathrm{~cm}^{-1}$ in ON resonance with the asymmetric band (001). (b) Zoomed image of S2(a) showing the same splitting for (001) in both TMM and experiment, and deviation in splitting for (101). (c) Zoomed image of S2(a) with changing the oscillator strength of (101) to match splitting in TMM with experiment. 
by observing the changes in the (101). For this purpose, $18 \mu \mathrm{m}$ pathlength cavity was prepared and $10^{\text {th }}$ mode of the cavity was ON resonantly coupled to the asymmetric stretching band (001). Simultaneously, the combination band (101) is also coupled to the $14^{\text {th }}$ cavity mode (Fig. S2(a)). TMM simulations were done for the same, and then a comparison of splitting energy from experimental value is done. After the correction, it was observed that for (001) band, splitting energy of approximately $280 \mathrm{~cm}^{-1}$ by both experimental TMM simulation. At the same time, the (101) band deviate approximately, $8 \mathrm{~cm}^{-1}$ with respect to experiment and TMM as shown in figure $\mathrm{S} 2 \mathrm{~b}$. This variation can be attributed to the change in the oscillator strength of (001) band.

In order to calculate the area ratio for the cavity experiments, the oscillator strength of the combination band (101) is arbitrarily decreased to get the same splitting energy as obtained from the experiment (Fig. S2(c)). After getting the same splitting energy for the combination band (101) in TMM, the mirrors were removed in the TMM calculations to obtain the IR spectrum under these cavity conditions. Thus, the value of area under combination band (101) obtained from the experiment deviates from that from TMM (figure S3). Since both TMM and experiment give the same value of splitting energy for the asymmetric band (001), so no changes were made to its oscillator strength; thus area under the curve remains the same for both TMM and experiment. The value of area ratio from the $\mathrm{ON}$ resonance cavity was 52.6, and the value predicted for the same without making any changes in the oscillator strength of (101) band was 44.6.

Table 2: Area ratio for different concentrations of $\mathrm{CS}_{2}$ in $\mathrm{ON}$ resonance cavity conditions.

\begin{tabular}{|c|c|c|c|}
\hline Sr. No. & Concentration of $\mathbf{C S}_{2}$ & Area Ratio in TMM & Area Ratio in experiments \\
\hline \hline 1 & $25 \%(4.14 \mathrm{M})$ & 44.32 & 46.43 \\
\hline 2 & $45 \%(7.45 \mathrm{M})$ & 44.27 & 48.15 \\
\hline 3 & $85 \%(14.07 \mathrm{M})$ & 44.40 & 50.8 \\
\hline 4 & $100 \%(16.55 \mathrm{M})$ & 44.67 & 52.49 \\
\hline
\end{tabular}

Similar area ratio calculations were done for different concentrations of $\mathrm{CS}_{2}$ in a $18 \mu \mathrm{m}$ thick cavity, and the result is summarized in Table 2. It is evident that for all the cases, the area ratio obtained in experiments deviates from the value as predicted from TMM. 


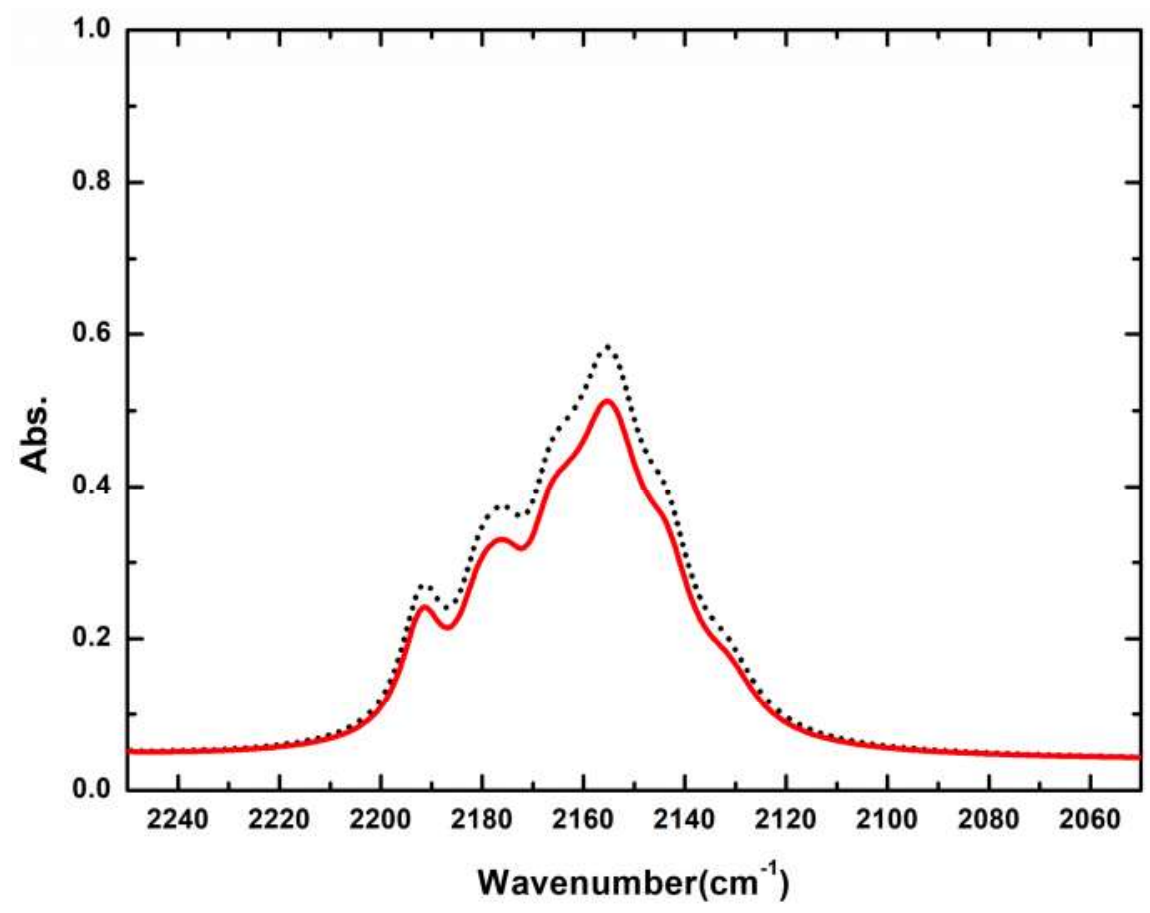

Figure $S 3$ : Absorption spectrum for (101) of pure $\mathrm{CS}_{2}$ in cavity conditions: TMM (Dotted black) and experiment (red).

\section{Area ratio in cavity tuning experiment:}

Cavity detuning is performed by decreasing the pathlength between the Au mirrors, in such a way that $10^{\text {th }}$ cavity mode slowly move away from ON resonance condition of (001) band (Figure S4).

Table 3: Area ratio deviation for the experiment from TMM in tuning experiments.

\begin{tabular}{|c|c|c|c|}
\hline Tuning Set & $\mathbf{1 0}^{\text {th }}$ mode & Area Ratio in TMM & Area Ratio in experiments \\
\hline \hline 1 (Black) & 1512.33 & 44.67 & 52.49 \\
\hline 2 (Red) & 1515.55 & 44.51 & 50.07 \\
\hline 3 (Green) & 1522.56 & 45.1 & 49 \\
\hline 4 (Blue) & 1529.19 & 45.10 & 48 \\
\hline
\end{tabular}




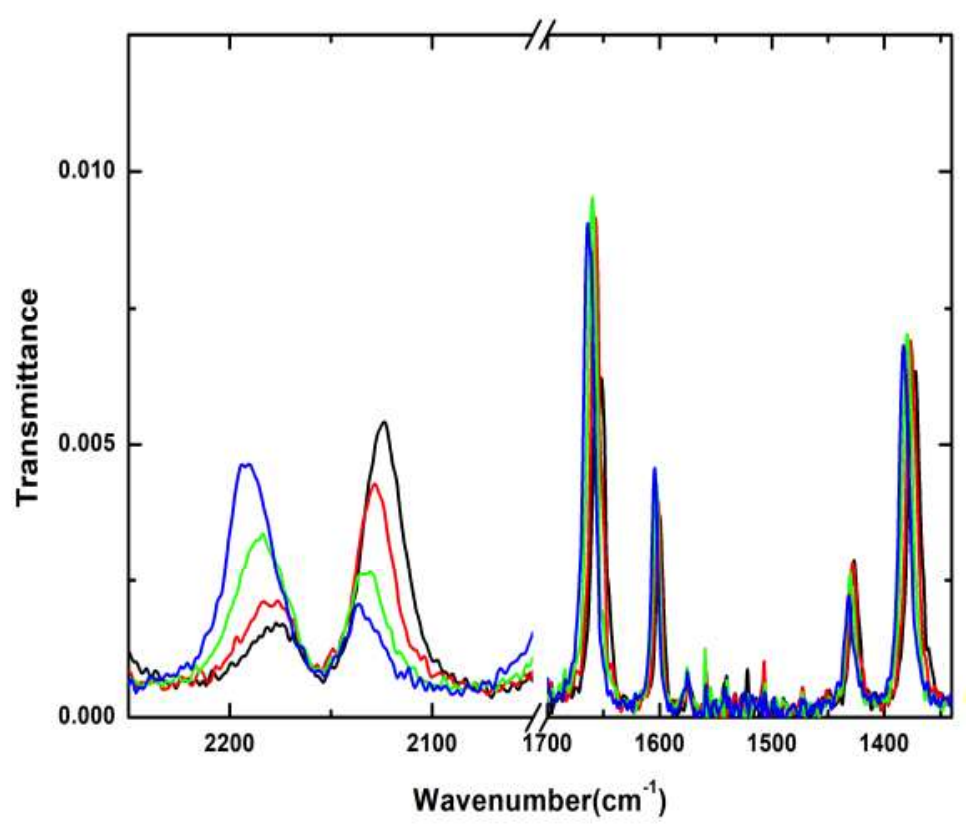

Figure $S 4$ : Cavity tuning experiment by physically varying the distance between the mirrors. Even though the cavity mode shifted from ON resonance condition to OFF resonance, still the coupling of asymmetric band (001) occurs as it is a very strong band. For each position of cavity mode in tuning experiment, TMM simulations were done, and the area ratio for both experiment and TMM compared as shown in Table 3. It is clear from Table 3 that as the cavity mode shifts from ON resonance condition to OFF resonance with respect to asymmetric band (001), the deviation of the experimental area ratio in TMM decreases.
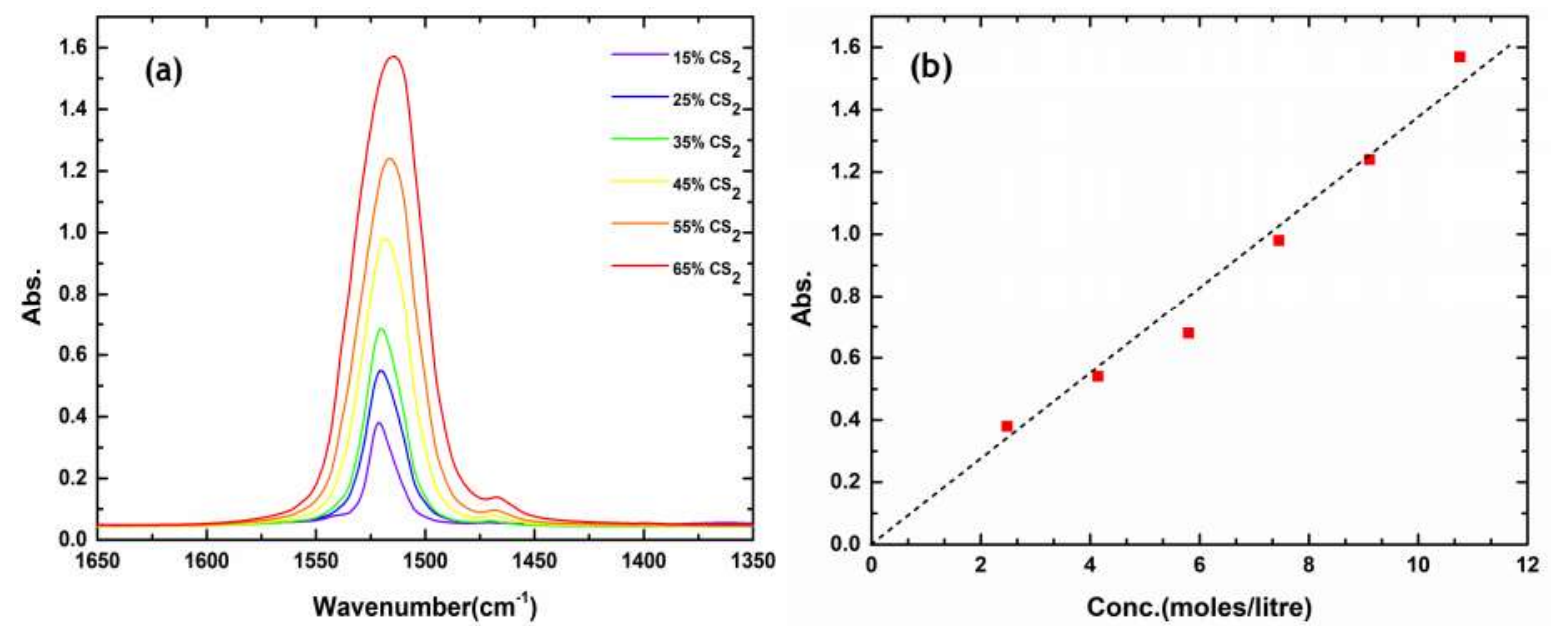

Figure S5: (a) Absorbance spectrum of (001) for different concentrations of $\mathrm{CS}_{2}$ in $\mathrm{CCl}_{4}$ in noncavity conditions. (b) The corresponding Beer-Lambert plot.

\section{Beer-Lambert plot for Asymmetric stretching band}

For understanding the self-interaction in non-cavities, varying concentration of $\mathrm{CS}_{2}$ in $\mathrm{CCl}_{4}$ was prepared and measured in $\mathrm{BaF}_{2}$ flow cell. A pathlength of $2 \mu \mathrm{m}$ is used for all the measurement 
and the corresponding IR spectrum was acquired in transmission configuration. Absorbance of (001) band is plotted against concentration as shown in Fig. S5 (a). This give a straight line indicating the system obeys Beer?s law and self-interactions are minimum for the non-cavity condition. Slope of the figure Fig. S5 (b) gives the molar absorptivity coefficient for asymmetric stretching band (001) as $684.85 \mathrm{~cm}^{-1} \mathrm{~mol}^{-1}$-lit.

\section{Theoretical expression for the area ratio}

The objective here is to derive the theoretical expression that is used in the main text for the area ratio between the fundamental band $(0,0,1)$ and the combination band $(1,0,1)$ of $\mathrm{CS}_{2}$ molecule. Here, area under the band is taken to be synonymous for all practical purposes to the intensity of the corresponding absorption band assuming Lorentzian profiles for each band with similar widths. We start with a recap of the basic quantum theory of linear triatomic molecules, following which an expression is obtained for the area/intensity ratio.

\subsection{The linear triatomic molecule}

The quantum theory of the linear triatomic molecule was worked out in detail in the early 1930s by Dennison, Fermi, Adel and others. ${ }^{1-4}$ We briefly outline this model before proceeding to examine the area ratio. We look at a generic molecule of the form $\mathrm{XY}_{2}$ with the coordinates of the two identical $\mathrm{Y}$ atoms given by $\left(x_{1}, y_{1}, z_{1}\right)$ and $\left(x_{2}, y_{2}, z_{2}\right)$ respectively. The coordinates of the central $\mathrm{X}$ atom is designated as $\left(x_{3}, y_{3}, x_{3}\right)$. The molecule has nine degrees of freedom out of which we are not interested in the three translation and two rotation degrees of freedom. Removing these five, we have four vibrational degrees of freedom that we focus on. We are taking $\mathrm{XY}_{2}$ to be linear like $\mathrm{CO}_{2}$ or $\mathrm{CS}_{2}$ and these vibrational degrees of freedom are readily identified as the symmetric stretching mode, the two out-of-plane bending modes ( 2 orthogonal directions) and the asymmetric bending mode. Without explicit diagonalization of the equations of motion and by defining the axis of the linear molecule as the $z$ axis, we can identify the corresponding normal mode coordinates as 
1. Corresponding to the symmetric stretching mode:

$$
q=\left(z_{1}-z_{2}\right)-a
$$

where $a$ is the equilibrium separation between the two $\mathrm{Y}$ atoms. This normal mode does not involve motion of the $\mathrm{X}$ atom.

2. Corresponding to the vibration of the $\mathrm{X}$ atom perpendicular to the molecular axis relative to the centre of mass of the two $\mathrm{Y}$ atoms that are assumed to be stationary, the relevant coordinates are:

$$
x=x_{3}-\frac{x_{1}+x_{2}}{2} \quad \text { and } \quad y=y_{3}-\frac{y_{1}+y_{2}}{2} .
$$

Utilising the symmetry of the molecule, we can as well use plane polar coordinates $r=$ $\sqrt{x^{2}+y^{2}}$ and $\phi=\tan ^{-1}(y / x)$ for the transverse vibrations.

3. Corresponding to the asymmetric mode of vibration wherein the $X$ atom moves along the molecular axis, the relevant normal mode cooridinate is

$$
z=z_{3}-\frac{z_{1}+z_{2}}{2}
$$

In identifying the normal mode coordinates we have assumed that the vibrational motion of the molecule is simple harmonic in nature and the corresponding potential are quadratic in the normal mode coordinates. These coordinates remain useful as long as anharmonicities in the potential are small and can be treated perturbatively.

In the harmonic regime, the Hamiltonian describing the vibrational motion can be written in terms of the normal mode coordinates ${ }^{4}$ as

$$
H_{0}=T_{0}+V_{0},
$$


where

$$
\begin{aligned}
T_{0} & =\frac{1}{2}(m / 2) \dot{q}^{2}+\frac{1}{2} \mu\left(\dot{r}^{2}+r^{2} \dot{\phi}^{2}\right)+\frac{1}{2} \mu \dot{z}^{2} \\
V_{0} & =\frac{1}{2}(m / 2) \omega_{1}^{2} q^{2}+\frac{1}{2} \mu \omega_{2}^{2} r^{2}+\frac{1}{2} \mu \omega_{3}^{2} z^{2} .
\end{aligned}
$$

In the expressions for the kinetic and potential energies above, $m$ is the mass of the $\mathrm{X}$ atom while

$$
\mu=\frac{2 m M}{m+2 M}
$$

is the reduced mass of the system with the $\mathrm{Y}$ atoms having mass $M$. The normal mode frequencies are $\omega_{1}, \omega_{2}$ and $\omega_{3}$ respectively.

A further change of coordinates is useful where we define

$$
\sigma=\sqrt{\frac{(m / 2) \omega_{1}}{\hbar}} q, \quad \rho=\sqrt{\frac{\mu \omega_{2}}{\hbar}} r, \quad \xi=\sqrt{\frac{\mu \omega_{3}}{\hbar}} z
$$

In terms of these scaled coordinates we have

$$
H_{0}=\frac{1}{2} \frac{\omega_{1}}{\hbar} p_{\sigma}^{2}+\frac{1}{2} \frac{\omega_{2}}{\hbar} p_{\rho}^{2}+\frac{1}{2} \frac{\omega_{2}}{\hbar \rho^{2}} p_{\phi}^{2}+\frac{1}{2} \frac{\omega_{3}}{\hbar} p_{\xi}^{2}+\frac{1}{2} \hbar \omega_{1} \sigma^{2}+\frac{1}{2} \hbar \omega_{2} \rho^{2}+\frac{1}{2} \hbar \omega_{3} \xi^{2}
$$

It is useful to introduce the following creation and annihilation operators,

$$
\begin{aligned}
a_{\sigma} & =\frac{1}{\sqrt{2}}\left(\sigma+\frac{i}{\hbar} p_{\sigma}\right) \quad ; & a_{\sigma}^{\dagger} & =\frac{1}{\sqrt{2}}\left(\sigma-\frac{i}{\hbar} p_{\sigma}\right), \\
a_{\xi} & =\frac{1}{\sqrt{2}}\left(\xi+\frac{i}{\hbar} p_{\xi}\right) \quad ; & a_{\xi}^{\dagger} & =\frac{1}{\sqrt{2}}\left(\xi-\frac{i}{\hbar} p_{\xi}\right), \\
a_{1} & =\frac{1}{\sqrt{2}}\left(\rho_{1}+\frac{i}{\hbar} p_{1}\right) \quad ; & a_{1}^{\dagger} & =\frac{1}{\sqrt{2}}\left(\rho_{1}-\frac{i}{\hbar} p_{1}\right), \\
a_{2} & =\frac{1}{\sqrt{2}}\left(\rho_{2}+\frac{i}{\hbar} p_{2}\right) \quad ; & a_{2}^{\dagger} & =\frac{1}{\sqrt{2}}\left(\rho_{2}-\frac{i}{\hbar} p_{2}\right) .
\end{aligned}
$$

where $\rho_{1}=\rho \cos \phi, \rho_{2}=\rho \sin \phi, p_{1}=\hbar \dot{\rho}_{1} / \omega_{2}$ and $p_{2}=\hbar \dot{\rho}_{2} / \omega_{2}$. We can now write the bare 
vibrational Hamiltonian $H_{0}$ as

$$
H_{0}=\hbar \omega_{1}\left(a_{\sigma}^{\dagger} a_{\sigma}+\frac{1}{2}\right)+\hbar \omega_{2}\left(a_{1}^{\dagger} a_{1}+a_{2}^{\dagger} a_{2}+1\right)+\hbar \omega_{3}\left(a_{\xi}^{\dagger} a_{\xi}+\frac{1}{2}\right)
$$

The eigenstates of $H_{0}$ can be labeled as $\left|n_{\sigma}, n_{\rho}^{l}, n_{\xi}\right\rangle$ for $n_{\sigma}, n_{\rho}, n_{\xi}=0,1, \ldots$, with $n_{\rho}=n_{1}+n_{2}$ and $l=n_{1}-n_{2}$, where $l=-n_{\rho},\left(-n_{\rho}+2\right), \ldots\left(n_{\rho}-2\right), n_{\rho}$. The corresponding energy eigenvalues are

$$
E_{n_{\sigma} n_{\rho} n_{\xi}}=\hbar \omega_{1}\left(n_{\rho}+\frac{1}{2}\right)+\hbar \omega_{2}\left(n_{\rho}+1\right)+\hbar \omega_{3}\left(n_{\xi}+\frac{1}{2}\right)
$$

and the corresponding wave functions are given by

$$
\Psi_{n_{\sigma}, n_{\rho}^{l}, n_{\xi}}(\sigma, \rho, \phi, \xi)=\psi^{n_{\sigma}}(\sigma) \psi^{n_{\xi}}(x) R^{n_{\rho}, l}(r) e^{i l \phi}
$$

where $\psi^{n}(x)$ are the usual Harmonic oscillator wave functions and $R^{n_{\rho}, l}(r) e^{i l \phi}$ are the eigenfunctions of the two-dimensional, isotropic Harmonic oscillator. Note that the energy eigenvalues are independent of the azimuthal quantum number $l$ indicating that the out-of-plane states have corresponding degeneracies.

In the realistic case, the molecular potentials are not harmonic. The variety of anharmonic terms that appear in the real potential are limited by considerations of the symmetry of the molecule. The anharmonic terms can be grouped into cubic and quartic ones and the Hamiltonian for the molecule can be written as

$$
H=H_{0}+\lambda H_{1}+\lambda^{2} H_{2}
$$

where $\lambda$ is an adjustable perturbation parameter and

$$
\begin{aligned}
\lambda H_{1} & =\lambda\left(a q^{3}+b q r^{2}+c q z^{2}\right) \\
\lambda^{2} H_{2} & =\lambda^{2}\left(d q^{4}+e r^{4}+f z^{4}+g q^{2} r^{2}+h q^{2} z^{2}+i r^{2} z^{2}\right),
\end{aligned}
$$

where $a, b, c, \ldots$ are constants that can be spectroscopically determined. We can define scaled 
coupling constants,

$$
A=a\left[\frac{(m / 2) \omega_{1}}{\hbar}\right]^{3 / 2}, \quad B=b \frac{\mu \omega_{2}}{\hbar} \sqrt{\frac{(m / 2) \omega_{1}}{\hbar}}, \text { etc. }
$$

and rewrite the anharmonic part of the Hamiltonian as

$$
\begin{aligned}
\lambda H_{1} & =\lambda\left(A \sigma^{3}+B \sigma \rho^{2}+C \sigma \xi^{2}\right) \\
\lambda^{2} H_{2} & =\lambda^{2}\left(D \sigma^{4}+E \rho^{4}+F \xi^{4}+G \sigma^{2} \rho^{2}+H \sigma^{2} \xi^{2}+I \rho^{2} \xi^{2}\right) .
\end{aligned}
$$

\subsection{Intensity of absorption}

The intensity of absorption from a state labelled by quantum numbers $\left(n_{\sigma}, n_{\rho}^{l}, n_{\xi}\right)$ to one labelled by $\left(\tilde{n}_{\sigma}, \tilde{n}_{\rho}^{\prime}, \tilde{n}_{\xi}\right)$ is given by, ${ }^{5}$

$$
I_{\tilde{n}_{\sigma}, \tilde{n}_{\rho}^{\prime}, \tilde{n}_{\xi}}^{n_{\sigma}, n_{\rho}^{l}, n_{\xi}}=2 \pi \frac{\omega}{3 c \hbar} \frac{\tilde{g}}{g} \sum_{j}\left|\left\langle\tilde{n}_{\sigma}, \tilde{n}_{\rho}^{l^{\prime}}, \tilde{n}_{\xi}\left|R_{j}\right| n_{\sigma}, n_{\rho}^{l}, n_{\xi}\right\rangle\right|^{2}\left(1-e^{-\hbar \omega / k T}\right)
$$

where $\omega$ is the frequency of the transition, $g$ and $\tilde{g}$ are the degeneracies of the initial and final states, $T$ is the temperature and $R_{j}$ are the components of the electric dipole moment of the molecule.

At room temperatures $\hbar \omega \gg k T$ and the thermal factor can be ignored in the above expressions. In the following we are interested in the transitions between the states with quantum numbers $\left(0,0^{0}, 0\right),\left(0,0^{0}, 1\right)$ and $\left(1,0^{0}, 1\right)$, all of whom have no degeneracy so that $\tilde{g}=g=1$. In particular we are interested in the ratio

$$
J=\frac{I_{0,0^{0}, 1}^{0,0^{0}, 0}}{I_{1,0^{0}, 1}^{0,0^{0}, 0}}=\frac{\omega_{001}}{\omega_{101}} \frac{\sum_{j}\left|\left\langle 0,0,0\left|R_{j}\right| 0,0,1\right\rangle\right|^{2}}{\sum_{j}\left|\left\langle 0,0,0\left|R_{j}\right| 1,0,1\right\rangle\right|^{2}}
$$

where we have suppressed the quantum number $l$ since for all the initial and final states we are considering, $l=0$ uniformly.

Only the asymmetric stretching mode and the bending mode of vibrations of the linear molecule contribute to the electric dipole moment. Given the axial symmetry of the molecule, we can write 
$\vec{R}=R_{r} \hat{r}+R_{z} \hat{z}$. Here we are assuming that the molecule does not have an intrinsic electric dipole moment and that the symmetric stretching mode displaces the charge distribution of the molecule in such a way that the net dipole moment remains zero. In other words, $R_{z}$ depends only on the normal mode coordinate $z$ and not on $q$. We can write

$$
R_{r}=\alpha^{\prime} r=\alpha \rho, \quad \text { and } \quad R_{z}=\beta^{\prime} z=\beta \xi
$$

where $\alpha^{\prime}$ and $\beta^{\prime}$ are constants specific to the molecule while $\alpha=\alpha^{\prime} \sqrt{\hbar / \mu \omega_{2}}$ and $\beta=\beta^{\prime} \sqrt{\hbar / \mu \omega_{3}}$. Since $n_{r}=l=0$ for all the three states we are interested in it is easy to show that $\left\langle R_{r}\right\rangle=0$ for both matrix elements appearing Eq. (10). Using $\xi=\left(a_{\xi}+a_{\xi}^{\dagger}\right) / \sqrt{2}$, we find that

$$
\left\langle\tilde{n}_{\sigma}, \tilde{n}_{\rho}^{l^{\prime}}, \tilde{n}_{\xi}\left|R_{\xi}\right| n_{\sigma}, n_{\rho}^{l}, n_{\xi}\right\rangle=\frac{\alpha}{\sqrt{2}} \delta_{\tilde{n}_{\sigma} n_{\sigma}} \delta_{\tilde{n}_{\rho} n_{\rho}} \delta_{l l^{\prime}} \delta_{\tilde{n}_{\xi} n_{\xi} \pm 1}
$$

It is easy to see that the denominator of Eq. (10) must vanish and the intensity ratio we are interested in must go to infinity. This is happening because in the absence of the anharmonic terms of the molecular Hamiltonian, the symmetric stretching mode cannot couple to the electromagnetic field at all. However, in reality the combination band $\left|1,0^{0}, 1\right\rangle$ is indeed observed and is found to be IR active in case of molecules like $\mathrm{CO}_{2}$ and $\mathrm{CS}_{2}$. This is due to the anharmonicities in the real potential function and the matrix elements that we should really be computing are $\left\langle\left\langle 0,0,0\left|R_{j}\right| 0,0,1\right\rangle\right\rangle$ and $\left\langle\left\langle 0,0,0\left|R_{j}\right| 1,0,1\right\rangle\right\rangle$ where the double kets, $\left.|\cdot\rangle\right\rangle$ denote the physical states that are the eigenstates of the full Hamiltonian, $H$.

\subsection{Physical states}

The anharmonic terms in $H$ can be treated as a perturbation on $H_{0}$. Note that the anharmonic terms in Section 7.1 or Section 7.1 are independent of $\phi$. This means that the perturbation cannot mix together eigenstates of $H_{0}$ with different values of $l$. So we can pick a value of $l$ and use nondegenerate perturbation theory instead of degenerate one despite the degeneracy that is otherwise there on account of the energy eigenvalues also not depending on the sign of $l$. The eigenvalues of 
$H$ can be readily written down to second order in perturbation as

$$
\begin{gathered}
E_{n_{\sigma} n_{r}^{l} n_{\xi}}=\text { Const }+n_{\sigma} \hbar \omega_{1}+n_{\rho} \hbar \omega_{2}+n_{\xi} \hbar \omega_{3}+x_{11} n_{\sigma}^{2}+x_{22}\left(n_{\rho}^{2}-l^{2} / 3\right) \\
+x_{33} n_{\xi}^{2}+x_{12} n_{\sigma} n_{\rho}+x_{13} n_{\sigma} n_{\xi}+x_{23} n_{\rho} n_{\xi}
\end{gathered}
$$

where $x_{i j}$ are constants that depend on the coupling constants $a, b, \ldots$ that appear in $\lambda H_{1}$ and $\lambda^{2} H 2$. First order perturbation, proportional to $\lambda$, to the energy is zero since the expectation values of all the cubic terms in $\lambda H_{1}$ are zero with respect to any of the eigenstates of $H_{0}$.

Non-degenerate perturbation theory gives the physical states as

$$
\left.\left|n_{\sigma}, n_{\rho}^{l}, n_{\xi}\right\rangle\right\rangle=\left|n_{\sigma}, n_{\rho}^{l}, n_{\xi}\right\rangle+\sum_{u v w} c_{u v w l}^{n_{\sigma} n_{r}^{l} n_{\xi}}\left|n_{\sigma}+u, n_{\rho}^{l}+2 v, n_{\xi}+2 w\right\rangle
$$

where $u, v, w$ are such that they are not equal to zero themselves but $n_{\sigma}+u, n_{\rho}+2 v$ and $n_{\xi}+2 w$ are all positive numbers with

$$
c_{u v w l}^{n_{\sigma} n_{r}^{l} n_{\xi}}=\frac{2 \pi}{u \omega_{1}+2 v \omega_{2}+2 w \omega_{3}}\left\langle n_{\sigma}+u, n_{\rho}^{l}+2 v, n_{\xi}+2 w\left|H^{\prime}\right| n_{\sigma}, n_{\rho}^{l}, n_{\xi}\right\rangle,
$$

where $H^{\prime}=\lambda H_{1}+\lambda^{2} H_{2}$. A long but straightforward computation yields,

$$
\begin{aligned}
\left.\left|0,0^{0}, 0\right\rangle\right\rangle=\left|0,0^{0}, 0\right\rangle+\lambda \frac{2 \pi q_{11}}{\omega_{1}}\left|1,0^{0}, 0\right\rangle+\lambda \frac{2 \pi q_{31}}{3 \omega_{1}}\left|3,0^{0}, 0\right\rangle \\
+\lambda \frac{2 \pi q_{32}}{\omega_{1}+2 \omega_{2}}\left|1,2^{0}, 0\right\rangle+\lambda \frac{2 \pi q_{33}}{\omega_{1}+2 \omega_{3}}\left|1,0^{0}, 2\right\rangle+\mathscr{O}\left(\lambda^{2}\right), \\
\left.\left|0,0^{0}, 1\right\rangle\right\rangle=\left|0,0^{0}, 1\right\rangle+\lambda \frac{2 \pi p_{11}}{\omega_{1}}\left|1,0^{0}, 1\right\rangle+\lambda \frac{2 \pi p_{31}}{3 \omega_{1}}\left|3,0^{0}, 1\right\rangle \\
+\lambda \frac{2 \pi p_{32}}{\omega_{1}+2 \omega_{2}}\left|1,2^{0}, 1\right\rangle+\lambda \frac{2 \pi p_{33}}{\omega_{1}+2 \omega_{3}}\left|1,0^{0}, 3\right\rangle+\mathscr{O}\left(\lambda^{2}\right),
\end{aligned}
$$


and

$$
\begin{aligned}
\left.\left|1,0^{0}, 1\right\rangle\right\rangle= & \left|1,0^{0}, 1\right\rangle+\lambda \frac{2 \pi s_{11}}{\omega_{1}}\left|0,0^{0}, 1\right\rangle+\lambda \frac{2 \pi s_{12}}{\omega_{1}}\left|2,0^{0}, 1\right\rangle+\lambda \frac{2 \pi s_{31}}{\omega_{1}+2 \omega_{2}}\left|0,2^{0}, 1\right\rangle \\
& +\lambda \frac{2 \pi s_{32}}{\omega_{1}+2 \omega_{2}}\left|2,2^{0}, 1\right\rangle+\lambda \frac{2 \pi s_{33}}{\omega_{1}+2 \omega_{3}}\left|0,0^{0}, 3\right\rangle+\lambda \frac{2 \pi s_{34}}{\omega_{1}+2 \omega_{3}}\left|2,0^{0}, 3\right\rangle+\mathscr{O}\left(\lambda^{2}\right),
\end{aligned}
$$

where $q_{i j}, p_{i j}$ and $s_{i j}$ are constants depending on the couplings in $H_{1}$ and $H_{2}$ and we have also ignored writing down explicitly the normalisation of the physical states.

In the expressions for $\left.\left.\left|0,0^{0}, 0\right\rangle\right\rangle,\left|0,0^{0}, 1\right\rangle\right\rangle$ and $\left.\left|1,0^{0}, 1\right\rangle\right\rangle$, we see that in all the terms $n_{\rho}$ is even. This means that $\left\langle\left\langle R_{r}\right\rangle\right\rangle=0$ continues to be zero when computed with respect to the physical states also because $R_{r}$ can only connect states which differ in $n_{\rho}$ by 1 . On the other hand if we compare the states $\left.\left|0,0^{0}, 0\right\rangle\right\rangle$ and $\left.\left|0,0^{0}, 1\right\rangle\right\rangle$ corresponding to the strongly IR-active fundamental of the asymmetric stretching mode of $\mathrm{CO}_{2}$ and $\mathrm{CS}_{2}$, we see that term-by-term, $n_{\xi}$ differs by one. Accounting for the normalisations of $\left.\left|0,0^{0}, 0\right\rangle\right\rangle$ and $\left.\left|0,0^{0}, 1\right\rangle\right\rangle$ also one can show that

$$
\left\langle\left\langle 0,0^{0}, 0\left|R_{z}\right| 0,0^{0}, 1\right\rangle\right\rangle \simeq\left\langle 0,0^{0}, 0\left|R_{z}\right| 0,0^{0}, 1\right\rangle=\beta,
$$

where $\beta$ is defined inEq. (11). Again, a straightforward computation yields,

$$
\left\langle\left\langle 0,0^{0}, 0\left|R_{z}\right| 1,0^{0}, 1\right\rangle\right\rangle=\mathscr{N}_{000} \mathscr{N}_{101} 2 \pi \beta \lambda\left(\frac{q_{11}}{\omega_{1}}+\frac{s_{11}}{\omega_{1}}+\frac{q_{33} \sqrt{2}}{\omega_{1}+2 \omega_{3}}\right),
$$

where $\mathscr{N}_{000}$ and $\mathscr{N}_{101}$ are the normalisations of the two physical states. Using Eq. (17) and Eq. (18) we get

$$
J=\frac{1}{4 \pi^{2} \lambda^{2} \mathscr{N}_{000}^{2} \mathscr{N}_{101}^{2}} \times \frac{\omega_{3}+x_{33}}{\omega_{1}+\omega_{3}+x_{11}+x_{33}+x_{13}} \times \frac{1}{\left(\frac{q_{11}}{\omega_{1}}+\frac{s_{11}}{\omega_{1}}+\frac{q_{33} \sqrt{2}}{\omega_{1}+2 \omega_{3}}\right)^{2}}
$$

We have used $\omega_{001}=\omega_{3}+x_{33}$ and $\omega_{101}=\omega_{1}+\omega_{3}+x_{11}+x_{33}+x_{13}$ incorporating corrections to the energies due to anharmonicity up to second order in $\lambda$. Note that in the third term on the right in Eq. (19) we have used only the bare frequencies $\omega_{1}$ and $\omega_{3}$ since the origin of these terms are 
from the perturbation series for the physical states. The constants $q_{11}, s_{11}$ and $q_{33}$ are proportional to $x_{11}, x_{33}$ and $x_{13}$ respectively.

\subsection{The $\mathrm{CS}_{2}$ Molecule}

Let us now specialise to the case of the Carbon Disulphide molecule. We are interested in computing the dependence of the area ratio between the strongly IR-active band corresponding to the fundamental of the asymmetric stretching mode and the weak $\left(1,0^{0}, 1\right)$ combination band. As mentioned earlier, the area under each band is taken to be proportional to the intensity of absorption (oscillator strength) and so the area ratio, apart from a proportionality constant is equal to the quantity $J$ for which we have obtained an expression for in Eq. (19). Under strong and ultra-strong coupling regimes the variable in Eq. (19) that has a dependence on the concentration of molecules in the cavity is the frequency $\omega_{3}$ of the asymmetric stretching mode and through this, the area ratio also acquires a dependence on the concentration.

For the $\mathrm{CS}_{2}$ molecule, the spectroscopically determined values of the relevant anharmonicity constants ${ }^{6}$ are $x_{11}=-0.7 \mathrm{~cm}^{-1}, x_{33}=-4.8 \mathrm{~cm}^{-1}$ and $x_{33}=-8.0 \mathrm{~cm}^{-1}$ while in the same units, the fundamentals of the symmetric and asymmetric stretching modes correspond to $655 \mathrm{~cm}^{-1}$ and $1510 \mathrm{~cm}^{-1}$ respectively. In our expression for $J$ we can therefore effectively ignore these constants relative to the frequencies $\omega_{1}$ and $\omega_{2}$. In addition to this, we note that the denominator of the third term in Eq. (19) also has a dependence on $\omega_{3}$ and consequently on the concentration. However this dependence is strongly suppressed by $q_{33}$ which, in turn, is proportional to $x_{33}$. For clarity of our discussion, we will choose to ignore the $\omega_{3}$ dependence of the denominator of the third term as well and write the area ratio as

$$
A=K J=\Gamma \frac{\omega_{3}}{\omega_{1}+\omega_{3}}
$$


where

$$
\Gamma=\frac{K}{4 \pi^{2} \lambda^{2} \mathscr{N}_{000}^{2} \mathscr{N}_{101}^{2}} \times \frac{1}{\left(\frac{q_{11}}{\omega_{1}}+\frac{s_{11}}{\omega_{1}}+\frac{q_{33} \sqrt{2}}{\omega_{1}+2 \omega_{3}}\right)^{2}},
$$

is assumed to be approximated well by a constant.

\section{Ultra-strong coupling to the cavity}

Let us consider $N$ molecules in the cavity. In the present case the $\left(0,0^{0}, 1\right)$ transition couples strongly with the $10^{\text {th }}$ harmonic of the cavity mode. We focus only on this cavity mode to the exclusion of all other modes that may be present. The cavity mode is described by the field operator,

$$
\vec{E}(\vec{r})=\sqrt{\frac{\hbar \omega_{c}}{2 \varepsilon_{0} V}}\left(a_{c} \vec{\varepsilon}_{\vec{k}} e^{-i \vec{k} \cdot \vec{r}}+a_{c}^{\dagger} \vec{\varepsilon}_{\vec{k}}^{*} e^{i \vec{k} \cdot \vec{r}}\right),
$$

where $\omega_{c}$ is the frequency of the relevant mode, $\vec{k}$ is its wave vector, $V$ is the mode volume, $a_{c}$ and $a_{c}^{\dagger}$ its corresponding creation and annihilation operators and $\vec{\varepsilon}_{\vec{k}}$ is the polarisation of the mode. In the present case the molecules are coupling to the vacuum of the electromagnetic field inside the cavity. We assume that the cavity mode has uniform intensity within the mode volume $V$ and that its polarisation is in the plane perpendicular to the axis of the cavity. The asymmetric stretching mode of those molecules whose axis lie on this plane would couple strongly to this mode while those with orientation perpendicular to this plane (along the axis of the cavity) will not couple at all. At intermediate orientation the coupling energy is modulated by the cosine of the angle that the molecular axis makes with the plane of polarisation of the mode. We are not concerned with the bending mode of vibration of the molecule here and assume that it is not resonant with any of the harmonics of the cavity mode.

For simplicity, we focus only on the $n$ molecules, out of the total $N$, whose axis is perpendicular to the axis of the cavity and aligned with the polarization direction of the cavity field. A more detailed computation relaxing this assumption can be done but it serves only to add unnecessary mathematical complexity to our treatment. We assume that the mode volume within which the 
collective excitations of the $n$ molecules we are interested in is quantized coincides with the volume of the cavity mode. With these simplifying assumptions, we can write the expression for the field operator in Eq. (21) as

$$
E=\sqrt{\frac{\hbar \omega_{c}}{2 \varepsilon_{0} V}}\left(a_{c}+a_{c}^{\dagger}\right)
$$

The induced dipole moment along the molecular axis due to the presence of the cavity field is

$$
\vec{p}(0)=\left[\frac{d\langle\vec{p}\rangle}{d z}\right]_{0} z=\left[\frac{d\langle\vec{p}\rangle}{d z}\right]_{0} \sqrt{\frac{\hbar}{\mu \omega_{3}}} \xi=\left[\frac{d\langle\vec{p}\rangle}{d z}\right]_{0} \sqrt{\frac{\hbar}{2 \mu \omega_{3}}}\left(a_{\xi}+a_{\xi}^{\dagger}\right)
$$

where $(d\langle\vec{p}\rangle / d z)_{0}$ arises from Taylor expanding the molecular polarizability about its equilibrium position. The collective polarization of the $n$ molecules is

$$
\vec{P}(0)=\sum_{j=1}^{n} \vec{p}(0)=\left[\frac{d\langle\vec{p}\rangle}{d z}\right]_{0} \sqrt{\frac{\hbar}{2 \mu \omega_{3}}} \sum_{j}\left(a_{\xi_{j}}+a_{\xi_{j}}^{\dagger}\right)
$$

The interaction between the cavity field and the molecules is given bu

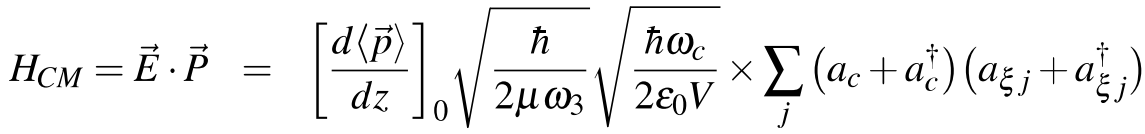

$$
\begin{aligned}
& =\hbar \Omega \sum_{j}\left(a_{c}+a_{c}^{\dagger}\right)\left(a_{\xi_{j}}+a_{\xi_{j}}^{\dagger}\right) \text {, }
\end{aligned}
$$

where

$$
\Omega=\left[\frac{d\langle\vec{p}\rangle}{d z}\right]_{0} \frac{1}{2 \sqrt{\mu \varepsilon_{0} V}}
$$

assuming that at resonance $\omega_{c}=\omega_{3}$. The coupling to the cavity field induces dipole moments in the molecules. The induced dipoles interact among each other and under ultra strong coupling, this interaction is also substantial and it cannot be ignored. The corresponding interaction Hamiltonian is

$$
H_{M M}=\frac{1}{2 \varepsilon_{0} V} \vec{P} \cdot \vec{P}=\left[\frac{d\langle\vec{p}\rangle}{d z}\right]_{0} \frac{\hbar}{4 \varepsilon_{0} \mu \omega_{3} V} \sum_{j, k}\left(a_{\xi_{j}}+a_{\xi_{j}}^{\dagger}\right)\left(a_{\xi_{k}}+a_{\xi_{k}}^{\dagger}\right)
$$




$$
=\hbar \frac{\Omega^{2}}{\omega_{3}} \sum_{j, k}\left(a_{\xi j}+a_{\xi_{j}}^{\dagger}\right)\left(a_{\xi_{k}}+a_{\xi_{k}}^{\dagger}\right) .
$$

The problem of vibrational modes strongly coupled to the IR field with the $H_{C M}$ and $H_{M M}$ terms included can be solved exactly using various approaches including the polariton transformation, Hopfield diagonalization etc. ${ }^{7-10}$ However our focus here is not on finding the eigenmodes and eigen-energies of the coupled system but rather on the dependence of the bare frequency of the asymmetric stretching mode $\omega_{3}$ on $n$ (and consequently on the concentration). For this, it is sufficient to take a semi-classical approach where we ignore the quantum features of the cavity field. The $\vec{E} \cdot \vec{P}$ coupling is linear in $\xi$ while the self interaction term is quadratic in $\xi$. Consider a general harmonic oscillator is suitably scaled coordinates with the Hamiltonian,

$$
h=\frac{1}{2} p^{2}+\frac{1}{2} \omega^{2} x^{2}
$$

Addition of a term of the form $k x^{2}$ can be treated easily and exactly since all it does is to shift the frequency to $\omega^{2}+k$. On the other hand, addition of a term $2 \omega^{2} c x$ can again be dealt with exactly by completion of the square shifting $x$ to $x+c$ without change in frequency of the oscillator but shifting the overall energy of the oscillator by a constant amount, namely $-c^{2} \omega^{2} / 2$. Here $c$ is a constant. This simple picture is consistent with our understanding of the cavity-molecule coupled dynamics in that the $\vec{E} \cdot \vec{P}$ term shifts the equilibrium value of $\xi$ thereby endowing each of the molecules a dipole moment along the direction of the electric field of the mode. These induced dipoles are significant in the resonant/ultra-strong coupling regime and they interact with each other leading to the $\vec{P} \cdot \vec{P}$ term that also needs to be treated exactly and not perturbatively.

Detailed theory of the molecule-cavity system ${ }^{8,9}$ shows that the effective coupling constant in $H_{C M}$ is given by $\hbar \Omega_{R}$ where $\Omega_{R}=\sqrt{n} \Omega$ is the Rabi frequency which is enhanced relative to the bare coupling $\Omega$ by the collective effect proportional to $\sqrt{n}$. The strength of the dipole-dipole coupling is similarly given by $\hbar \Omega_{R}^{2} / \omega_{3}=2 n \hbar \Omega^{2} / \omega_{3}$. We see that the bare frequency of the asymmetric 
stretching mode behaves as

$$
\omega_{3}^{(n)}=\omega_{3}+2 n \frac{\Omega^{2}}{\omega_{3}^{2}} \equiv \omega_{3}+\kappa n .
$$

We choose a simple form for $\omega_{1} \omega_{3}^{(n)}$ appearing in the denominator Eq. (20) as

$$
\omega_{1}+\omega_{3}^{(n)}=\omega_{1}+\omega_{3}+\eta \kappa n
$$

So finally we arrive at the following expression for the concentration dependence of the area ratio:

$$
\begin{aligned}
A & =\Gamma \frac{\omega_{3}+\kappa n}{\omega_{1}+\omega_{3}+\eta \kappa n}, \\
& =\Gamma \frac{\omega_{3}}{\omega_{1}+\omega_{3}} \frac{1+n \kappa / \omega_{3}^{2}}{1+n \eta \kappa /\left(\omega_{1}+\omega_{3}\right)}, \\
& =A_{0} \frac{1+k_{1} C}{1+k_{2} C}
\end{aligned}
$$

where $A_{0}=\Gamma \omega_{3} /\left(\omega_{1}+\omega_{3}\right)$ is the area ratio in the absence of the cavity. In the last line of the equation above we have replaced $n$ with the concentration $C$ of $\mathrm{CS}_{2}$ injected into the cavity with $C=\gamma n$ and the constants $k_{1}=\gamma \kappa / \omega_{3}$ and $k_{2}=\eta \gamma \kappa /\left(\omega_{1}+\omega_{3}\right)$.

\section{References}

(1) Adel, A.; Dennison, D. M. The Infrared Spectrum of Carbon Dioxide. Part I. Physical Review 1933, 43, 716-723.

(2) Adel, A.; Dennison, D. M. The Infrared Spectrum of Carbon Dioxide. Part II. Physical Review 1933, 44, 99-104.

(3) Dennison, D. M. The Vibrational Levels of Linear Symmetrical Triatomic Molecules. Physical Review 1932, 41, 304-312.

(4) Dennison, D. M. The Infrared Spectra of Polyatomic Molecules Part I. Reviews of Modern Physics 1931, 3, 280-345. 
(5) Tolman, R. C. Statistical Mechanics With Applications to Physics and Chemistry; The Chemical Catalog Company: New York, 1927.

(6) Ribni Kar, S. V.; Putzić, O. S. The infrared spectrum of carbon disulphide in liquid and dissolved states: anharmonicity of the carbon disulphide molecule in the liquid. Spectrochimica Acta Part A: Molecular Spectroscopy 1973, 29, 307-317.

(7) George, J.; Chervy, T.; Shalabney, A.; Devaux, E.; Hiura, H.; Genet, C.; Ebbesen, T. W. Multiple Rabi Splittings under Ultrastrong Vibrational Coupling. Phys. Rev. Lett. 2016, 117, 153601.

(8) Ciuti, C.; Bastard, G.; Carusotto, I. Quantum vacuum properties of the intersubband cavity polariton field. Physical Review B 2005, 72, 115303.

(9) Hopfield, J. J. Theory of the Contribution of Excitons to the Complex Dielectric Constant of Crystals. Physical Review 1958, 112, 1555-1567.

(10) Chervy, T.; Thomas, A.; Akiki, E.; Vergauwe, R. M. A.; Shalabney, A.; George, J.; Devaux, E.; Hutchison, J. A.; Genet, C.; Ebbesen, T. W. Vibro-Polaritonic IR Emission in the Strong Coupling Regime. ACS Photonics 2018, 5, 217-224. 